\title{
GEOMETRIC CHARACTERIZATIONS OF FINITE CHEVALLEY GROUPS OF TYPE $\mathrm{B}_{2}$
}

\author{
KOEN THAS AND HENDRIK VAN MALDEGHEM
}

\begin{abstract}
Finite Moufang generalized quadrangles were classified in 1974 as a corollary to the classification of finite groups with a split BN-pair of rank 2, by P. Fong and G. M. Seitz (1973), (1974). Later on, work of S. E. Payne and J. A. Thas culminated in an almost complete, elementary proof of that classification; see Finite Generalized Quadrangles, 1984. Using slightly more group theory, first W. M. Kantor (1991), then the first author (2001), and finally, essentially without group theory, J. A. Thas (preprint), completed this geometric approach. Recently, J. Tits and R. Weiss classified all (finite and infinite) Moufang polygons (2002), and this provides a third independent proof for the classification of finite Moufang quadrangles.

In the present paper, we start with a much weaker condition on a BNpair of Type $\mathbf{B}_{2}$ and show that it must correspond to a Moufang quadrangle, proving that the BN-pair arises from a finite Chevalley group of (relative) Type $\mathbf{B}_{2}$. Our methods consist of a mixture of combinatorial, geometric and group theoretic arguments, but we do not use the classification of finite simple groups. The condition on the BN-pair translates to the generalized quadrangle as follows: for each point $x$, the stabilizer of all lines through that point acts transitively on the points opposite $x$.
\end{abstract}

\section{INTRODUCTION}

Generalized quadrangles arise as the natural geometries associated with Chevalley groups/algebraic groups/classical groups/mixed groups of (relative) Type $\mathbf{B}_{2}$. All these groups carry the structure of a BN-pair, in the sense of Tits [36]. In fact, roughly speaking, BN-pairs with a Weyl group of Type $\mathbf{B}_{2}$ are essentially equivalent with generalized quadrangles admitting a certain homogeneity property (in the terminology and notation below: transitivity on ordered quadruples $\left(z_{1}, z_{2}, z_{3}, z_{4}\right)$ of points with $z_{1} \sim z_{2} \sim z_{3} \sim z_{4} \sim z_{1}$ and $\left.z_{1} \not z_{3} ; z_{2} \not z_{4}\right)$.

Finite BN-pairs of Type $\mathbf{B}_{2}$ (in fact, more generally, of rank 2) can be classified using the classification of finite simple groups; see [4]. However, it remains interesting to prove such classifications without invoking the classification of finite simple groups, mainly because so-called "elementary proofs" usually give much more information about the geometric or group theoretical structure under investigation. Moreover, new ideas needed in the proof could be used in different situations.

Received by the editors January 18, 2005 and, in revised form, December 28, 2005.

2000 Mathematics Subject Classification. Primary 05B25, 51E12, 20B10, 20B25, $20 \mathrm{E} 42$.

Key words and phrases. (Split) BN-pair, Chevalley group, Moufang condition, generalized quadrangle.

The first author is a Postdoctoral Fellow of the Fund for Scientific Research — Flanders (Belgium). 
In the present paper we are interested in classifying generalized quadrangles that satisfy a weak variant of the Moufang property for finite generalized quadrangles, or, equivalently, a weak form of the notion of a split BN-pair of Type $\mathbf{B}_{2}$, without invoking the classification of finite simple groups. It is not surprising that we have to appeal to not-so-elementary group theoretic work such as the classification of finite split BN-pairs of rank 1.

Recall that a group $G$ is said to have a $B N$-pair $(B, N)$, where $B, N$ are subgroups of $G$, if the following properties are satisfied:

(TI1) $\langle B, N\rangle=G$;

(TI2) $H=B \cap N \triangleleft N$ and $N / H=W$ is a Coxeter group with distinct generators $s_{1}, s_{2}, \ldots, s_{n}$

(TI3) $B s_{i} B w B \subseteq B w B \cup B s_{i} w B$ whenever $w \in W$ and $i \in\{1,2, \ldots, n\}$;

(TI4) $s_{i} B s_{i} \neq B$ for all $i \in\{1,2, \ldots, n\}$.

The subgroup $B$, respectively $W$, is a Borel subgroup, respectively the Weyl group, of $G$. The natural number $n$ is called the rank of the BN-pair; in this paper, the rank (which corresponds to the rank of the associated building) is always 2 . In that case, the Weyl group $N /(B \cap N)$ is a dihedral group of size $2 m$ for some $m$. We say that the BN-pair $(B, N)$ is of Type $\mathbf{B}_{2}$ if $W$ is a dihedral group of size 8 . The associated building mentioned above is the incidence structure with elements the cosets of the maximal subgroups containing $B$, and two (distinct) such cosets being incident if their intersection is nonempty. There is no loss of generality to assume that $G$ acts faithfully on that structure, and we will do so throughout. It is well known that there are precisely $n$ maximal subgroups containing $B$, and they are called the maximal parabolic subgroups, or maximal parabolics.

Let $G$ be a group with a BN-pair $(B, N)$ of Type $\mathbf{B}_{2}$, and let $P_{1}, P_{2}$ be the two maximal parabolic subgroups containing $B$. For $i=1,2$, let $s_{i} \in G$ normalize $N$ and $P_{i}$, but not $P_{3-i}$. Put $H=B \cap N$, as usual. We introduce Condition ( $\dagger$ ).

$(\dagger)$ For some $i \in\{1,2\}$, there exists a subgroup $U$ of $B$ with $U \unlhd P_{i}$ such that $U H=B \cap B^{s_{i}}$.

We can now state our Main Result. We recall that we assume that a group with a BN-pair acts faithfully on the associated building. Also, a group $G$ with a BN-pair $(B, N)$ is split if there is a normal nilpotent subgroup $U$ of $G$ with $B=U(B \cap N)$.

Main Result. A finite group $G$ with a BN-pair of Type $\mathbf{B}_{2}$ satisfying $(\dagger)$ is isomorphic to an almost simple group with socle one of the simple classical groups $\mathbf{S}_{4}(q) \cong \mathbf{O}_{5}(q), \mathbf{U}_{4}(q) \cong \mathbf{O}_{6}^{-}(q)$ or $\mathbf{U}_{5}(q)$, for some prime power $q$. Hence for $a$ group $G$ with a BN-pair of Type $\mathbf{B}_{2}$ the conditions $(\dagger)$ and "being split" are equivalent.

In order to prove that result, we translate this condition to the corresponding building related to the group $G$, and this building is a so-called "generalized quadrangle" (for an abstract definition, see below), whose elements are "points" and "lines". We say that a point $x$ of a generalized quadrangle $\mathcal{S}$ is a center of transitivity if there is a group of collineations fixing all lines through $x$ and acting transitively on the points not on a common line with $x$. A direct translation (see below) of our Main Result yields:

Main Result (Geometric Version). If in a finite generalized quadrangle $\mathcal{S}$ every point $x$ is a center of transitivity, then $\mathcal{S}$ is a classical generalized quadrangle related 
to the standard BN-pair in one of the classical groups mentioned in the Main Result above (for an explicit list see Section 2.4 below).

We use a mixture of geometric, combinatorial and group theoretic arguments to prove the Geometric Version of our Main Result. In fact, it is rather fascinating to see how all these methods add up to deliver a complete proof. If geometric or combinatorial methods fall short, then group theory helps out; conversely, where group theory would need long and highly nontrivial and technical arguments, geometry and combinatorics provide short and elegant solutions. Moreover, we have to distinguish many cases, and all of them use different results in geometry, combinatorics or group theory. Although we do not know of any counterexample, we do not see how to extend our proof to the infinite case.

In order to make our proof more transparent, we have organized it as follows. We first prove a weaker assertion (we assume that the quadrangle is what we call half pseudo Moufang). With an eye on generalization to other types of rank 2 BN-pairs, this deserves separate mention anyway. From this weaker assertion, we derive our Main Result with some additional arguments.

Our Main Result implies the solution of some long-standing conjectures and open problems. The most famous is the following: prove without the classification of finite simple groups that every finite thick generalized quadrangle, every point of which is an elation point, is a classical (or dual classical) generalized quadrangle. We refer to Theorem C in Section 3.

In the next section we introduce generalized quadrangles and we mention some important basic facts. In Section 3 we translate Condition $(\dagger)$ to geometry, and we introduce our intermediate condition which we call the half pseudo Moufang condition. Sections 4 up to 7 are devoted to the classification of half pseudo Moufang generalized quadrangles. In Section 8, we prove our Main Result (Geometric Version).

\section{Finite Generalized QUADRANGLeS}

2.1. Finite generalized quadrangles: combinatorics. A (finite) generalized quadrangle $(G Q)$ of order $(s, t), s, t \in \mathbb{N}^{*}$, is an incidence structure $\mathcal{S}=(\mathcal{P}, \mathcal{L}, \mathrm{I})$ in which $\mathcal{P}$ and $\mathcal{L}$ are disjoint (nonempty) sets of objects called points and lines respectively, and for which $I$ is a symmetric point-line incidence relation satisfying the following axioms:

(1) Each point is incident with $t+1$ lines $(t \geq 1)$ and two distinct points are incident with at most one line.

(2) Each line is incident with $s+1$ points $(s \geq 1)$ and two distinct lines are incident with at most one point.

(3) If $x$ is a point and $L$ is a line not incident with $x$, then there is a unique point-line pair $(y, M)$ such that $x \mathrm{I} M \mathrm{I} y \mathrm{I} L$.

If $s=t$, then $\mathcal{S}$ is also said to be of order $s$. If $s>1$ and $t>1$, then we call the GQ thick. Generalized quadrangles were introduced by Tits in his celebrated work on triality [34] as a subclass of a larger class of incidence structures, namely the generalized polygons, in order to provide a geometric interpretation of the Chevalley groups of rank 2. The main results up to 1983 on finite generalized quadrangles are contained in the monograph Finite Generalized Quadrangles (denoted by "FGQ" throughout) by Payne and [J. A.] Thas [17]. 
There is a point-line duality for GQ's of order $(s, t)$ for which in any definition or theorem the words "point" and "line", and the parameters $s$ and $t$, are interchanged. Normally, we assume without further notice that the (point-line) dual of a given theorem or definition has also been given.

Let $\mathcal{S}=(\mathcal{P}, \mathcal{L}, \mathrm{I})$ be a (finite) thick generalized quadrangle of order $(s, t)$. Then $|\mathcal{P}|=(s+1)(s t+1)$ and $|\mathcal{L}|=(t+1)(s t+1)$. Also, $s \leq t^{2}[9,10]$ and, dually, $t \leq s^{2}$. We will refer to these basic inequalities as "The inequalities of Higman".

If $\mathcal{S}$ is a GQ of order $(s, 1)$, then $\mathcal{S}$ is also called a grid with parameters $s+1, s+1$. Dual grids are defined dually. A flag of a GQ is an incident point-line pair. An anti-flag is a nonincident point-line pair. If $(p, L)$ is an anti-flag of the GQ $\mathcal{S}$, then by $\operatorname{proj}_{L} p$ we denote the unique point on $L$ which is collinear with $p$. Dually we define the notion $\operatorname{proj}_{p} L$.

Let $x$ and $y$ be (not necessarily distinct) points of the GQ $\mathcal{S}$ of order $(s, t)$; we write $x \sim y$ and say that $x$ and $y$ are collinear, provided that there is some line $L$ such that $x \mathrm{I} L \mathrm{I} y$ (and $x \nsim y$ means that $x$ and $y$ are not collinear). Dually, for $L, M \in \mathcal{L}$, we write $L \sim M$ or $L \not M$ according to whether $L$ and $M$ are concurrent or nonconcurrent. If $x \neq y \sim x$, the line incident with both $x$ and $y$ is denoted by $x y$, and if $L \sim M \neq L$, the point which is incident with both $L$ and $M$ is sometimes denoted by $L \cap M$. For $x \in \mathcal{P}$, we put $x^{\perp}=\{y \in \mathcal{P} \| y \sim x\}$ (note that $x \in x^{\perp}$ ). For a pair of distinct points $\{x, y\}$, the trace of $\{x, y\}$ is defined as $x^{\perp} \cap y^{\perp}$, and we denote this set by $\{x, y\}^{\perp}$. Then $\left|\{x, y\}^{\perp}\right|=s+1$ or $t+1$, according to whether $x \sim y$ or $x \not y$, and in the latter case, the trace is called nontrivial. More generally, if $A \subseteq \mathcal{P}, A^{\perp}$ is defined by $A^{\perp}=\bigcap_{x \in A} x^{\perp}$. For $x \neq y$, the span of the pair $\{x, y\}$ is $\{x, y\}^{\perp \perp}=\left\{z \in \mathcal{P} \| z \in u^{\perp}\right.$ for all $\left.u \in\{x, y\}^{\perp}\right\}$ (so $\left.\{x, y\}^{\perp \perp}=\left(\{x, y\}^{\perp}\right)^{\perp}\right)$. Then $\left|\{x, y\}^{\perp \perp}\right|=s+1$ or $\left|\{x, y\}^{\perp \perp}\right| \leq t+1$ according to whether $x \sim y$ or $x \not y$.

If $x \sim y, x \neq y$, or if $x \not y$ and $\left|\{x, y\}^{\perp \perp}\right|=t+1$, we say that the pair $\{x, y\}$ is regular. The point $x$ is regular provided $\{x, y\}$ is regular for every $y \in \mathcal{P} \backslash\{x\}$. One easily proves that either $s=1$ or $t \leq s$ if $\mathcal{S}$ has a regular pair of noncollinear points; see Chapter 1 of FGQ. Regularity for lines is defined dually.

A triad of points is a triple of pairwise noncollinear points. Given a triad $T$, a center of $T$ is just an element of $T^{\perp}$.

2.2. Subquadrangles. A subquadrangle, or also $\operatorname{sub} G Q, \mathcal{S}^{\prime}=\left(\mathcal{P}^{\prime}, \mathcal{L}^{\prime}, \mathrm{I}^{\prime}\right)$ of a $\mathrm{GQ}$ $\mathcal{S}=(\mathcal{P}, \mathcal{L}, \mathrm{I})$ is a GQ for which $\mathcal{P}^{\prime} \subseteq \mathcal{P}, \mathcal{L}^{\prime} \subseteq \mathcal{L}$, and where $\mathrm{I}^{\prime}$ is the restriction of I to $\left(\mathcal{P}^{\prime} \times \mathcal{L}^{\prime}\right) \cup\left(\mathcal{L}^{\prime} \times \mathcal{P}^{\prime}\right)$. The following well known result will be used, often without notice.

Theorem 2.1 (2.2.2 of FGQ). Let $\mathcal{S}^{\prime}$ be a proper subquadrangle of the $G Q \mathcal{S}$, where $\mathcal{S}$ has order $(s, t)$ and $\mathcal{S}^{\prime}$ has order $\left(s, t^{\prime}\right)$ (so $\left.t>t^{\prime}\right)$. Then we have

(1) $t \geq s$; if $s=t$, then $t^{\prime}=1$.

(2) If $s>1$, then $t^{\prime} \leq s$; if $t^{\prime}=s \geq 2$, then $t=s^{2}$.

(3) If $s>1$ and $t^{\prime}>1$, then $\sqrt{s} \leq t^{\prime} \leq s$ and $s^{3 / 2} \leq t \leq s^{2}$.

(4) If $t=s^{3 / 2}>1$ and $t^{\prime}>1$, then $t^{\prime}=\sqrt{s}$.

(5) Let $\mathcal{S}^{\prime}$ have a proper subquadrangle $\mathcal{S}^{\prime \prime}$ of order $\left(s, t^{\prime \prime}\right), s>1$. Then $t^{\prime \prime}=1$, $t^{\prime}=s$ and $t=s^{2}$.

If $\mathcal{S}^{\prime}$ is a subGQ with order $\left(s, t^{\prime}\right)$ of the GQ $\mathcal{S}$ with order $(s, t)$, and $x$ is a point of $\mathcal{S}$ not in $\mathcal{S}^{\prime}$, then clearly, on every line of $\mathcal{S}^{\prime}$, there is a unique point of $\mathcal{S}^{\prime}$ collinear with $x$. This set of points forms a so-called ovoid of $\mathcal{S}^{\prime}$ (i.e., a set $1+s t^{\prime}$ mutually 
noncollinear points of $\mathcal{S}^{\prime}$ ), which is called the ovoid subtended by $x$. The dual of an ovoid is a spread, and a subtended spread is defined dually in a subquadrangle with order $\left(s^{\prime}, t\right)$.

2.3. Automorphisms. A collineation or automorphism of a generalized quadrangle $\mathcal{S}=(\mathcal{P}, \mathcal{L}, \mathrm{I})$ is a permutation of $\mathcal{P} \cup \mathcal{L}$ which preserves $\mathcal{P}, \mathcal{L}$ and I.

Let $L$ be a line of a thick GQ $\mathcal{S}$ of order $(s, t)$. A symmetry about $L$ is an automorphism of $\mathcal{S}$ which fixes every element of $L^{\perp}$. The line $L$ is called an axis of symmetry if the group $H$ of symmetries about $L$ has size $s$. In such a case, if $M \in L^{\perp} \backslash\{L\}$, then $H$ acts regularly on the set of points of $M$ not incident with $L$. Dually, one defines the notion of center of symmetry. It is easy to see that every axis of symmetry is a regular line. A whorl about $L$ is an automorphism of $\mathcal{S}$ fixing all points incident with $L$. An elation about the line $L$ is a whorl about $L$ that fixes no line in the set of lines not concurrent with $L$. By definition, the identity is an elation about every line. The line $L$ is called an elation line if there is a group of elations about $L$ acting (sharply) transitively on the set of lines not concurrent with $L$. The line $L$ is called a translation line if it is an elation line and if every point incident with $L$ is a regular point. This is equivalent with requiring that $L$ is an elation line and that the corresponding group of elations is abelian; see $[27,17]$. Finally, the line $L$ is called an axis of transitivity if the group of whorls about $L$ acts transitively on the set of lines not concurrent with $L$. Dually, one defines whorls, elations about a point, elation points, translation points and centers of transitivity.

The following theorem will often be used without further notice.

Theorem 2.2 (2.4.1 of FGQ). Let $\theta$ be an automorphism of the $G Q \mathcal{S}=(\mathcal{P}, \mathcal{L}, \mathrm{I})$ of order $(s, t)$. The substructure $\mathcal{S}_{\theta}=\left(\mathcal{P}_{\theta}, \mathcal{L}_{\theta}, \mathrm{I}_{\theta}\right)$ of $\mathcal{S}$ which consists of the fixed elements of $\theta$ must be given by (at least) one of the following:

(i) $\mathcal{L}_{\theta}=\emptyset$ and $\mathcal{P}_{\theta}$ is a set of pairwise noncollinear points;

(i) ${ }^{\prime} \mathcal{P}_{\theta}=\emptyset$ and $\mathcal{L}_{\theta}$ is a set of pairwise nonconcurrent lines;

(ii) $\mathcal{P}_{\theta}$ contains a point $x$ so that $y \sim x$ for each $y \in \mathcal{P}_{\theta}$, and each line of $\mathcal{L}_{\theta}$ is incident with $x$;

(ii) ${ }^{\prime} \mathcal{L}_{\theta}$ contains a line $L$ so that $M \sim L$ for each $M \in \mathcal{L}_{\theta}$, and each point of $\mathcal{P}_{\theta}$ is incident with $L$;

(iii) $\mathcal{S}_{\theta}$ is a grid;

(iii) $^{\prime} \mathcal{S}_{\theta}$ is a dual grid;

(iv) $\mathcal{S}_{\theta}$ is a subGQ of $\mathcal{S}$ of order $\left(s^{\prime}, t^{\prime}\right), s^{\prime}, t^{\prime} \geq 2$.

2.4. Classical and dual classical generalized quadrangles. We now briefly describe the thick finite classical GQ's. They are related to the classical (Chevalley) groups (of Type $\mathbf{B}_{2}$ ).

(i) Consider a nonsingular quadric of Witt index 2, that is, of projective index 1 , in $\mathbf{P G}(4, q)$ and $\mathbf{P G}(5, q)$, respectively. The points and lines of the quadric form a generalized quadrangle which is denoted by $\mathcal{Q}(4, q)$ and $\mathcal{Q}(5, q)$, respectively, and has order $(q, q)$ and $\left(q, q^{2}\right)$, respectively. The corresponding simple Chevalley groups are $\mathbf{O}_{5}(q)$ and $\mathbf{O}_{6}^{-}(q)$, respectively.

(ii) Next, let $\mathcal{H}$ be a nonsingular Hermitian variety in $\mathbf{P G}\left(3, q^{2}\right)$, respectively PG $\left(4, q^{2}\right)$. The points and lines of $\mathcal{H}$ form a generalized quadrangle $H\left(3, q^{2}\right)$, respectively $H\left(4, q^{2}\right)$, which has order $\left(q^{2}, q\right)$, respectively $\left(q^{2}, q^{3}\right)$. The simple Chevalley groups are the unitary groups $\mathbf{U}_{4}(q)$ and $\mathbf{U}_{5}(q)$, respectively. 
(iii) The points of $\mathbf{P G}(3, q)$ together with the totally isotropic lines with respect to a symplectic polarity form a GQ $W(q)$ of order $q$. The corresponding Chevalley group is the symplectic group $\mathbf{S}_{4}(q)$.

For more details and properties of these classical quadrangles, see Chapter 3 of FGQ. We just note that the dual of $W(q)$ is isomorphic to $\mathcal{Q}(4, q)$, and that the dual of $H\left(3, q^{2}\right)$ is isomorphic to $\mathcal{Q}(5, q)$, which can also be seen from the groups, as $\mathbf{O}_{5}(q) \cong \mathbf{S}_{4}(q)$ and $\mathbf{O}_{6}^{-}(q) \cong \mathbf{U}_{4}(q)$. The point-line duals of the classical GQ's are called the dual classical GQ's.

\section{Half PSEudo Moufang QuAdrangles AND The MAin Results}

A panel of a GQ $\mathcal{S}=(\mathcal{P}, \mathcal{L}, \mathrm{I})$ is an element $(x, L, y)$ of $\mathcal{P} \times \mathcal{L} \times \mathcal{P}$ for which $x \mathrm{I} L \mathrm{I} y$ and $x \neq y$. Dually, one defines dual panels. A Moufang generalized quadrangle is a $\mathrm{GQ} \mathcal{S}=(\mathcal{P}, \mathcal{L}, \mathrm{I})$ in which the following conditions hold:

(M) for any panel $(x, L, y)$ of $\mathcal{S}$, the group $H$ of all automorphisms of $\mathcal{S}$ fixing all lines incident with $x$, all lines incident with $y$, and all points incident with $L$ (sometimes called root-elations), acts transitively on the set of points that are incident with some given line $M \mathrm{I} x, M \neq L$, and different from $x$;

$\left(\mathrm{M}^{\prime}\right)$ for any dual panel $(L, x, M)$ of $\mathcal{S}$, the group of all automorphisms of $\mathcal{S}$ fixing all points incident with $L$, all those incident with $M$, and all lines incident with $x$ (also called root-elations), acts transitively on the set of lines that are incident with a given point $z \mathrm{I} L, z \neq x$, and different from $L$.

Remark 3.1. If Condition (M) holds in a thick GQ $\mathcal{S}$, then, with the notation of (M), if $M^{\prime} \neq L$ is an arbitrary line through $x$, respectively $y$, then $H$ also acts transitively on the points incident with $M^{\prime}$, different from $x$, respectively $y$. The same remark holds for dual panels.

Let $\mathcal{S}$ be a thick Moufang GQ. The group generated by all root-elations will be called the little projective group.

Moufang quadrangles were introduced in the appendix of [37] (as a special case of Moufang spherical buildings). It was noted by Tits in [38] that the classification of finite split BN-pairs of rank 2 by Fong and Seitz [5,6] (or rather a corollary of their main result; see Section 5.7 of [43] for a detailed discussion) implies that a thick finite generalized quadrangle is Moufang if and only if it is a classical or dual classical GQ (but at present there is also the monograph [40] by Tits and Weiss where all Moufang polygons are classified). In [25], [J. A.] Thas, Payne and the second author proved that the Properties $(\mathrm{M})$ and $\left(\mathrm{M}^{\prime}\right)$ are equivalent. Hence, if we call a GQ half Moufang if it satisfies (M) or $\left(\mathrm{M}^{\prime}\right)$, then every half Moufang finite GQ is Moufang, and hence classical or dual classical. We will use this result in the rest of the paper a few times.

Now let $\mathcal{S}=(\mathcal{P}, \mathcal{L}, \mathrm{I})$ be a thick GQ of order $(s, t)$ and let $H$ be an automorphism group of $\mathcal{S}$. For points $x, y$ of $\mathcal{S}$, we denote by $H_{x, y}$ the stabilizer of both $x$ and $y$ in $H$. Similarly for lines. Consider the following conditions:

(PM) For every panel $(x, L, y)$, with $x, y \in \mathcal{P}$ and $L \in \mathcal{L}$, there is a normal subgroup $H(x, y)$ of $H_{x, y}$ of elations about both $x$ and $y$ which acts regularly on the set of points that are incident with any line $M I x$, respectively $M^{\prime} \mathrm{I} y$, $M \neq L \neq M^{\prime}$, and different from $x$, respectively $y$. The group $H(x, y)$ will sometimes be referred to as a pseudo elation group. 
$\left(\mathrm{PM}^{\prime}\right)$ For every dual panel $(L, x, M)$, with $L, M \in \mathcal{L}$ and $x \in \mathcal{P}$, there is a normal subgroup $H(L, M)$ of $H_{L, M}$ of elations about both $L$ and $M$ which acts regularly on the set of lines that are incident with an arbitrary point $y \mathrm{I} L$, respectively $z \mathrm{I} M, y \neq x \neq z$, and different from $L$, respectively $M$.

A GQ is called pseudo Moufang (with respect to $H$ ) if both Properties (PM) and $\left(\mathrm{PM}^{\prime}\right)$ hold. It is called half pseudo Moufang if one of the Properties (PM) or $\left(\mathrm{PM}^{\prime}\right)$ holds. The (half) pseudo Moufang condition was, under a slightly different form, introduced by the first author during some lectures in the Seminar of Incidence Geometry at Ghent University in 1999 [26] in order to study the so-called 'strong elation generalized quadrangles' (see below).

Throughout, we will sometimes write 'HPMGQ' instead of 'half pseudo Moufang generalized quadrangle' for the sake of convenience, and we always denote the corresponding group by $H$. Hence we will often forget to mention this group explicitly.

Let $(x, L, y)$ be a panel of the thick generalized quadrangle $\mathcal{S}=(\mathcal{P}, \mathcal{L}, \mathrm{I})$ of order $(s, t)$ and suppose that $s<t$. Let $S(x, y)$ be the set of elations about both $x$ and $y$, and suppose that $|S(x, y)| \geq s$. Suppose moreover that $S(x, y)$ is not a group. Then clearly there is some automorphism $\phi \neq \mathbf{1}$ of $\mathcal{S}$ generated by $S(x, y)$ which fixes all lines incident with $x$ and all lines incident with $y$, and which fixes some point of $\mathcal{S}$ not incident with $L$. By Theorem 2.2, the set of fixed points and lines of $\phi$ forms a subGQ of $\mathcal{S}$ of order $\left(s^{\prime}, t\right), s^{\prime}<s$, contradicting the fact that $s<t$ (cf. Theorem 2.1). Hence if $s<t$, then Property (PM) is equivalent with requiring that for every panel $(x, L, y)$, there is a set of $s$ elations about both $x$ and $y$ all contained in $H$.

It is easy to see that in a pseudo Moufang GQ every point is a center of transitivity and every line is an axis of transitivity. Finite GQ's every point of which is a center of transitivity and every line of which is an axis of transitivity are classified in [42] (and all such GQ's are classical or dual classical). Consequently every finite pseudo Moufang GQ is a Moufang GQ. It is now our first aim to classify the thick finite half pseudo Moufang GQ's. The following result will be obtained.

Theorem A. Every finite thick half pseudo Moufang generalized quadrangle is a classical or dual classical quadrangle (or, equivalently, a Moufang quadrangle). Conversely, every (finite) classical or dual classical generalized quadrangle (or, equivalently, every finite Moufang quadrangle) is a half pseudo Moufang quadrangle with respect to any group $H$ containing the little projective group, and the pseudo elation groups are independent of $H$. In particular, every pseudo elation group is a group of root-elations.

Extending the terminology of [42], we will call a quadrangle each point of which is a center of transitivity, or each line of which is an axis of transitivity, a half 2-Moufang GQ. The following is a reformulation of the Main Result (Geometric Version).

Theorem B. Every finite half 2-Moufang generalized quadrangle is classical or dual classical.

A generalized quadrangle is called a strong elation generalized quadrangle (SEGQ) if each of its points is an elation point. It is a well known open problem to classify the SEGQ's without the classification of finite simple groups (see Payne [15, 16], K. Thas [28] and Van Maldeghem [42]). As each point of a half 
Moufang quadrangle (satisfying Property $(M)$ ) is an elation point, see Chapter 9 of FGQ, this problem also generalizes the problem considered in [25].

A consequence of Theorem $\mathrm{B}$ reads as follows:

Theorem C. Each finite strong elation generalized quadrangle is classical or dual classical.

We now prove the equivalence of Condition $(\dagger)$ with the condition of a half 2-Moufang quadrangle, giving rise to our Main Result.

First assume $(\dagger)$. Let $\mathcal{S}$ be the corresponding GQ. Without loss of generality, we may assume $i=1$ and $P_{1}$ corresponds to a point $x$ (which is stabilized by $\left.P_{1}\right)$; the parabolic $P_{2}$ then stabilizes a line $L$. The stabilizer of the flag $\{x, L\}$ is the Borel subgroup $B$. Since $U$ stabilizes $L$, and $U$ is normal in $P_{1}$ (which acts transitively on the lines through $x$ ), it is easy to see that $U$ stabilizes every line through $x$. Since $H$ fixes some point of $\mathcal{S}$ not collinear with $x$ (namely, the unique point not collinear with $x$ in the orbit of $x$ under the action of $N$ ), and since $B \cap B^{s_{1}}$ acts transitively on the points not collinear with $x$ (by the BN-pair property), we conclude that $U H=B \cap B^{s_{1}}$ implies that $U$ also acts transitively on the set of points not collinear with $x$. So $x$ is a center of transitivity.

Conversely, a standard argument (along the lines of the first paragraph of the proof of Lemma 4.1) shows that a (thick) half 2-Moufang GQ corresponds to a group $G$ with a BN-pair of Type $\mathbf{B}_{2}$. Taking for $U$ the full group of whorls (in $G$ ) about the point $x$ corresponding to the Borel subgroup $B$, we easily see that $U$ satisfies Condition $(\dagger)$ for $P_{1}$ the stabilizer of $x$.

We now mention an additional consequence of our Main Result (Geometric Version).

\section{Theorem D. A finite $G Q \mathcal{S}$ satisfying}

(UM) for any panel $(p, L, q)$ of $\mathcal{S}$, and any line $M \mathrm{I} q, M \neq L$, the group of all automorphisms of $\mathcal{S}$ fixing all lines incident with $p$ and fixing $M$, acts transitively on the set of points incident with $M$, and different from $q$,

is classical or dual classical.

Conversely, every classical or dual classical GQ satisfies (UM).

Indeed, suppose that $\mathcal{S}$ satisfies Property $(\mathrm{UM})$. For $(p, L, q)$ an arbitrary panel of $\mathcal{S}$, and $M \neq L$ an arbitrary line through $q$, denote the group as defined by (UM) by $H(p, L, q ; M)$. Fix the point $p$, and define the group $\Phi(p)$ by

$$
\Phi(p)=\langle H(p, L, q ; M) \| q \sim p \neq q, M \mathrm{I} q, M \neq L\rangle .
$$

Then $\Phi(p)$ is a group of whorls about $p$. By a result of Cuypers mentioned and proved in [3], the natural geometry $\Gamma(p)$ defined by $\mathcal{P} \backslash p^{\perp}$ (and thus having lineset $\mathcal{L} \backslash\{N \| N I p\})$ is connected. So, for each two distinct points $x$ and $y$ of $\Gamma(p)$, there is a set of distinct points $\left\{x=z_{0}, z_{1}, \ldots, z_{r}=y\right\}$ for some $r \in \mathbb{N}_{0}$, so that $z_{i} \sim z_{i+1}$ for $i=0,1, \ldots, r-1$. Clearly, for each such $i$, there is a collineation $\theta_{i}$ in $\Phi(p)$ mapping $z_{i}$ onto $z_{i+1}$, hence we have that $\Phi(p)$ acts transitively on $\mathcal{P} \backslash p^{\perp}$. By Theorem B, the first assertion of Theorem D follows.

Now suppose $p$ is a center of transitivity of a GQ $\mathcal{S}$. Then the following is easy to observe: for any panel of the form $(p, L, q)$, and any line $M I q, M \neq L$, the group of all automorphisms of $\mathcal{S}$ fixing all lines incident with $p$ and fixing $M$, acts transitively on the set of points incident with $M$, and different from $q$. Since in 
a classical generalized quadrangle every point is a center of transitivity, the final assertion of Theorem D follows.

In particular, it now follows that in Theorem A, we may delete from Condition (PM) the assumption that $H(x, y)$ is a normal subgroup, and we may replace the condition of $H(x, y)$ acting regularly by acting transitively, and we still end up with the same conclusion.

Standing Hypothesis. For the sake of simplicity, we will from now on use the notion of a half pseudo Moufang GQ only for GQ's satisfying Property (PM).

\section{Finite Moufang quadrangles are pseudo Moufang Quadrangles}

Here, we prove the second part of Theorem A, namely, that every classical and dual classical GQ is an HPMGQ "in a unique way". We first show a general lemma and use the notation of the previous section.

Lemma 4.1. If $\mathcal{S}$ is an $H P M G Q$ with respect to the group $H$, then $H$ acts transitively on the set of ordered quadruples $\left(z_{1}, z_{2}, z_{3}, z_{4}\right)$ of points with $z_{1} \sim z_{2} \sim z_{3} \sim$ $z_{4} \sim z_{1}$ and $z_{1} \nsim z_{3} ; z_{2} \nsim z_{4}$.

Proof. Let $L, M, M^{\prime}$ be three distinct lines of $\mathcal{S}$ with $M \nsim L \nsim M^{\prime}$. We claim that some element of $H$ fixes $L$ and maps $M$ to $M^{\prime}$.

First suppose that $M^{\prime} \sim M$. Let $x=\operatorname{proj}_{L}\left(M \cap M^{\prime}\right)$ and let $y$ be an arbitrary point on $L$ distinct from $x$. Select a nontrivial $\theta \in H(x, y)$ and denote $M^{\prime \prime}=M^{\theta}$. Choose any line $M_{0}$ meeting both $M^{\prime}$ and $M^{\prime \prime}$, but not incident with $x$, and put $L^{\prime}=\operatorname{proj}_{x} M_{0}$. Set $y^{\prime}=M_{0} \cap L^{\prime}$. Then there exists $\theta^{\prime} \in H\left(x, y^{\prime}\right)$ mapping $M^{\prime \prime}$ to $M^{\prime}$. Hence we found $\sigma=\theta \theta^{\prime} \in H$ fixing $L$ and mapping $M$ to $M^{\prime}$.

Next suppose that $M \nsim M^{\prime}$. Then either $L \in\left\{M, M^{\prime}\right\}^{\perp \perp}$ — in which case there exists for every pair of distinct points $x, y$ incident with $L$, a collineation $\sigma \in H(x, y)$ mapping $M$ to $M^{\prime}$ — or there exists a line $M^{*}$ meeting both $M$ and $M^{\prime}$ and not meeting $L$. Applying the foregoing argument twice, our claim follows. We now easily conclude that $H$ acts transitively on the set of ordered nonconcurrent pairs of lines.

Hence, for two quadruples $\left(z_{1}, z_{2}, z_{3}, z_{4}\right)$ and $\left(z_{1}^{\prime}, z_{2}^{\prime}, z_{3}^{\prime}, z_{4}^{\prime}\right)$ as in the statement of the lemma, we may assume $z_{1} z_{2}=z_{1}^{\prime} z_{2}^{\prime}$ and $z_{3} z_{4}=z_{3}^{\prime} z_{4}^{\prime}$. Applying elements of $H(x, y)$, for appropriate points $x, y$ such that $z_{1} z_{2} \mathrm{I} x \sim y \mathrm{I} z_{3} z_{4}$, the result follows easily.

Remark 4.2. Completely similar, one also shows that in a half 2-Moufang GQ the full automorphism group acts transitively on ordered pairs of opposite points (lines).

Proposition 4.3. Let $\mathcal{S}$ be a finite Moufang (and hence classical or dual classical) quadrangle with little projective group $H$. Then $\mathcal{S}$ is an $H P M G Q$ with respect to $H$ if one defines the pseudo elation groups as the corresponding root-elation groups. Moreover, if $\mathcal{S}$ is half pseudo Moufang with respect to some other group $H^{*}$, then $H \leq H^{*}$ and the pseudo elation groups are necessarily the root-elation groups.

Proof. The first statement is obvious and follows from the semi-regular action of any root-elation. Suppose now that the classical or dual classical GQ $\mathcal{S}$ is half pseudo Moufang with respect to the group $H^{*}$. By Lemma 4.1, $H^{*}$ is flag-transitive and hence it contains all root-elations, except possibly if $\mathcal{S}$ is isomorphic to $W(2)$, $W(3), \mathcal{Q}(4,2), \mathcal{Q}(4,3)$ or $H(3,9)$ (this is due to Seitz [18]; it is also mentioned in 
Theorem 4.8.7 of [43]). But it is easily seen that in the first four cases the elements of any pseudo elation group must be root-elations. If $\mathcal{S}$ is isomorphic to $H(3,9)$ and if $H^{*}$ does not contain all root-elations of $\mathcal{S}$, then it follows from [18] that $H^{*}$ has order at most $4|\mathbf{P S L}(3,4)|=2^{8} .3^{2} .5 .7$, which is much less than the number of ordered quadruples of points (as in the statement of Lemma 4.1) in $H(3,9)$ (that number is equal to $2^{5} .3^{6} .5 .7$ ), contradicting the fact that $H^{*}$ acts transitively on the set of such ordered quadruples of $\mathcal{S}$. Hence we have shown that $H^{*}$ contains all root-elations of $\mathcal{S}$ and consequently that $H \leq H^{*}$.

Now let the order of $\mathcal{S}$ be equal to $(s, t)$ and let $\theta \in H^{*}(x, y)$, for some distinct collinear points $x, y$. Note that there exists a root-elation $\sigma$ fixing all lines through $x$, all lines through $y$, and such that $z^{\theta}=z^{\sigma}$ for at least one point $z$ not incident with $x y$. The automorphism $\theta \sigma^{-1}$ fixes pointwise a subquadrangle of order $\left(s^{\prime}, t\right)$, with $1<s^{\prime} \leq s$. Indeed, $1<s^{\prime}$ because $\theta$ is an elation and hence its order divides the prime power $s$, which implies that it fixes at least three points of the line $x y$. If $s \leq t$, then necessarily $s=s^{\prime}$ by Theorem 2.1, and so $\theta=\sigma$. That leaves two cases.

(1) $\mathcal{S}$ IS DUAL TO $H(4, t)$. We have $H(x, y) \unlhd H_{x, y}^{*}$, so every Sylow $p$ subgroup $P$ of $H_{x, y}^{*}$, where $p$ is the unique prime number dividing $s$ and $t$, contains $H(x, y)$ (which, we recall, consists of root-elations). We choose $P$ such that it contains $\theta$. Write $(s, t)=\left(p^{3 k}, p^{2 k}\right)$. If $\theta \notin H(x, y)$, then $\theta \sigma^{-1}$ fixes pointwise a subquadrangle $\mathcal{S}^{\prime}$ of order $\left(s^{\prime}, p^{2 k}\right)$. By 2.2.1 of FGQ, $s^{\prime} p^{2 k} \leq p^{3 k}$, hence $s^{\prime} \leq p^{k}$. So $s^{\prime}=p^{k}$ by the inequality of Higman, and $\mathcal{S}^{\prime}$ is dual to $H(3, t)$ (so isomorphic to $\mathcal{Q}(5, \sqrt{t})$ ). Now $\theta \sigma^{-1}$, which belongs to $P$, must act trivially on the set of lines through $x$. So, interpreted in $\mathbf{P G}(4, t)$ (by dualizing to $H(4, t)$ ), the collineation $\theta \sigma^{-1}$ is linear. If we now interpret the set of points incident with $x y$ as a Hermitian curve in a classical projective plane of order $t$, then $\theta \sigma^{-1}$ is a linear automorphism of that curve fixing at least two points of the curve, and hence also fixing the "polar point" of the joining line of those two fixed points. Hence $\theta \sigma^{-1}$ fixes a triangle in that projective plane, implying that its order divides $(t-1)^{2}$ (remembering that $\theta \sigma^{-1}$ is linear) and therefore cannot be contained in $P$, a contradiction.

(2) $\mathcal{S}$ Is IsOmorphic to $H(3, s)$. In this case, it is easily seen that $H^{*}(x, y)$, for distinct points $x \sim y$, must contain at least one root-elation $\sigma$ (otherwise a Sylow $p$-subgroup ( $p$ as before) of $H_{x, y}^{*}$ would have size at least

$$
\left|\left\langle H(x, y), H^{*}(x, y)\right\rangle\right|=s^{2},
$$

as $H \leq H^{*}$ and $H(x, y) \unlhd H_{x, y}^{*}$, a contradiction). Let $L$ be any line through $x$ distinct from $x y$. Then $H_{L}$ acts on $L$ as $\mathbf{P S L}_{2}(s)$; see e.g. [43, Table 8.1]. It is not so hard to see that $H_{L, x}$ has the same action on the points incident with $L$ as $H_{L, x, y}$. So we may conjugate $\sigma$ by $H_{L, x, y}$ and obtain, if $s$ is even, all root-elations of $H(x, y)$, or, if $s$ is odd, $\frac{s-1}{2}$ elements of $H(x, y)$, which generate $H(x, y)$. Consequently, since $H^{*}(x, y)$ is normal in $H_{x, y}^{*}$, we conclude $H^{*}(x, y)=H(x, y)$.

We prove the first part of Theorem A in the next sections. We start with some general observations, which will in particular settle the case $s>t$. We then invoke the classification of finite split BN-pairs of rank 1 and consider the case of sharply 2 -transitive groups first. The other cases are treated afterwards. Whenever we can, we state lemmas and propositions in a more general context. 


\section{Some General observations}

5.1. Property $(\mathbf{H})$. The first properties that we will observe only rely on the fact that a half pseudo Moufang GQ is a half 2-Moufang GQ. So we first show this.

Lemma 5.1. Suppose the thick $G Q \mathcal{S}=(\mathcal{P}, \mathcal{L}, \mathrm{I})$ of order $(s, t)$ is half pseudo Moufang. Then every point of $\mathcal{S}$ is a center of transitivity.

Proof. $\quad$ Suppose $x$ is an arbitrary point of $\mathcal{S}$. Let $H(x)$ be the group defined by $\langle H(x, y) \| y \sim x, y \neq x\rangle$; then it is clear that every element of $H(x)$ fixes every line incident with $x$. It is also clear, by the definition of $H(x)$, that if $y$ and $z$ are distinct collinear points in $\mathcal{P} \backslash x^{\perp}$, then there is an element of $H(x)$ mapping $y$ onto $z$.

Now suppose $y$ and $z$ are noncollinear points in $\mathcal{P} \backslash x^{\perp}$. By an observation first made by Cuypers (see Brouwer [3]), there exists a sequence of points $y=$ $u_{0}, u_{1}, \ldots, u_{\ell}=z$ with $u_{i-1} \sim u_{i}$ and $u_{i} \nsim x$, for all $i \in\{1,2, \ldots, \ell\}$. Applying the first part of the proof $\ell$ times, the lemma follows.

Definition 5.2. Now let $x$ and $y$ be two noncollinear points of the GQ $\mathcal{S}=$ $(\mathcal{P}, \mathcal{L}, \mathrm{I})$. Then we put $\operatorname{cl}(x, y)=\left\{z \in \mathcal{P} \| z^{\perp} \cap\{x, y\}^{\perp \perp} \neq \emptyset\right\}$. A point $u$ has Property (H) provided that $z \in \operatorname{cl}(x, y)$ if and only if $y \in \operatorname{cl}(x, z)$ whenever $(x, y, z)$ is a triad of points in $u^{\perp}$; we also denote the dual notion by Property $(\mathrm{H})$.

Lemma 5.3. Suppose $\mathcal{S}$ is a thick $G Q$ of order $(s, t)$ such that some point $u$ of $\mathcal{S}$ is a center of transitivity. Then $u$ has Property $(\mathrm{H})$.

Proof. Let $\{x, y, z\}$ be a triad in $u^{\perp}$ with the property that $z \in \operatorname{cl}(x, y)$. Suppose $v$ is a point such that $v \sim z$ and $v \in\{x, y\}^{\perp \perp}$. Consider a whorl $\phi$ about $u$ which maps $v$ onto $z$, and which fixes $x$ (such a whorl exists by the transitivity assumption). Then $y^{\phi} \sim y$ and $z=v^{\phi} \in\left\{x, y^{\phi}\right\}^{\perp \perp}$, and hence $y \in \operatorname{cl}(x, z)$. It now easily follows that the point $u$ has Property $(\mathrm{H})$.

Isomorphic generalized quadrangles are defined in the usual standard way. We use the notation $\mathcal{S} \cong \mathcal{S}^{\prime}$ to denote isomorphic quadrangles $\mathcal{S}$ and $\mathcal{S}^{\prime}$.

Proposition 5.4. Suppose every point of the thick $G Q \mathcal{S}$ of order $(s, t)$ is a center of transitivity. Then one of the following possibilities occurs:

(a) $\mathcal{S}$ is classical (and isomorphic to $W(s)$ or $H(4, s)$ ), or

(b) every span of noncollinear points has size 2 , or

(c) $s>t$ and every point is regular.

In particular, this applies to half pseudo Moufang generalized quadrangles.

Proof. By Lemma 5.3 each point has Property (H). Now apply 5.6.2 of FGQ, noting that from 5.2.1 of FGQ follows that a (thick) finite generalized quadrangle of order $(s, t)$ with $s \leq t$ and all points regular is necessarily isomorphic to $W(s)$.

5.2. Some results on elation generalized quadrangles. If $s \leq t$, then there is a further restriction on the structure of an HPMGQ of order $(s, t)$, which can be stated in the (implicitly) more general context of elation GQ's. Its proof is based on 9.5.1 of FGQ (see also [26]).

Proposition 5.5. Let $\mathcal{S}=(\mathcal{P}, \mathcal{L}, \mathrm{I})$ be a thick $G Q$ of order $(s, t)$ and suppose $x \in \mathcal{P}$ is an elation point with elation group $G$. Suppose that for every $y \in \mathcal{P} \backslash x^{\perp}$ the span $\{x, y\}^{\perp \perp}$ has exactly 2 elements, and that for every line LIx and every 
line $M \in \mathcal{L} \backslash L^{\perp}$ the span $\{L, M\}^{\perp \perp}$ has exactly 2 elements. Then the center of $G$ is trivial. In particular, $s>t$.

Proof. Denote the lines through $x$ by $L_{i}, i \in\{0,1, \ldots, t\}$, and let $y$ be an arbitrary point of $\mathcal{P} \backslash x^{\perp}$. Put $M_{i}:=\operatorname{proj}_{y} L_{i}$ for every $i$, and let $G_{i}$ be the stabilizer of $M_{i}$ in $G$ (so $G_{i}$ acts regularly on the points of $M_{i}$ distinct from $L_{i} \cap M_{i}$ ). Furthermore, let $G_{i}^{*}$ be the stabilizer of $x_{i}:=M_{i} \cap L_{i}$ in $G$. Suppose by way of contradiction that the center $Z(G)$ of $G$ is nontrivial and let $g$ be a nontrivial element of $Z(G)$. If $g \in \bigcap_{i=0}^{s} G_{i}^{*}$, then $\left\{y, y^{g}, x\right\} \subseteq\{x, y\}^{\perp \perp}$, a contradiction since $y^{g} \neq y$. Hence, without loss of generality, we may assume that $g$ does not belong to $G_{0}^{*}$. Since $G_{0}$ centralizes $g$, the group $G_{0}$ fixes $M_{0}^{g}$. The latter line does not meet $M_{0}$. Let $M_{j}$ be the unique line through $y$ which meets $M_{0}^{\prime}=M_{0}^{g}$. Then we conclude that every element of the orbit of $M_{j}$ under the action of $G_{0}$ meets every element of the triad $\left\{M_{0}, M_{0}^{\prime}, L_{j}\right\}$. Since that orbit has size $s$ and also $L_{0}$ is a center of the triad, we see that $\left\{M_{0}, M_{0}^{\prime}, L_{j}\right\} \subseteq\left\{M_{0}, L_{j}\right\}^{\perp \perp}$, again a contradiction. Hence $Z(G)$ is trivial.

Suppose now that $s \leq t$. Then by Frohardt [7], $s$ and $t$ are powers of the same prime number $p$, and hence $G$ is a $p$-group. But every such group has a nontrivial center.

The proposition is proved.

If an HPMGQ is also an EGQ, then we can prove Property $(\mathrm{H})$ for lines.

Lemma 5.6. Let $\mathcal{S}$ be a thick $H P M G Q$ of order $(s, t)$ such that for each point $x$ the group $H(x)=\langle H(x, y) \mid y \sim x \neq y\rangle$ is a group of elations about $x$. Then each line of $\mathcal{S}$ satisfies Property $(\mathrm{H})$.

Proof. Let $V, W$ be two opposite lines. Denote the points on $V$ and $W$ by $v_{i}$ and $w_{i}, i \in\{0,1, \ldots, s\}$, respectively. We can choose the indices in such a way that $v_{i} \sim w_{i}$, and we denote the line $v_{i} w_{i}$ by $L_{i}, i \in\{0,1, \ldots, s\}$. We prove Property (H) for the line $L_{0}$. It suffices to prove that, if $Z \in\{V, W\}^{\perp \perp} \backslash\{V, W\}$, and if $W^{\prime}$ is an arbitrary line through $w_{0}$, then for every $i \in\{2,3, \ldots, s\}$, there exists a line $Z^{\prime}$ concurrent with $\operatorname{proj}_{v_{1}} W^{\prime}$ and with $\operatorname{proj}_{v_{i}} W^{\prime}$ and incident with the point $z:=Z \cap L_{0}$. Here, the lines $V, W, Z$ play the role of the points $x, y, z$, respectively, in Definition 5.2.

Let $\theta \in H\left(v_{0}, w_{0}\right)$ map $v_{1}$ onto $v_{i}$. Let $\theta^{\prime} \in H\left(v_{0}, z\right)$ map $v_{i}$ onto $v_{1}$. Then $\theta \theta^{\prime} \in H\left(v_{0}\right)$ and $\theta \theta^{\prime}$ fixes $L_{1}$. It follows that $\theta \theta^{\prime} \in H\left(v_{0}, v_{1}\right)$. Hence $\theta \theta^{\prime}$ fixes $L^{\prime}:=\operatorname{proj}_{v_{1}} W^{\prime}$. Since $\theta$ fixes $W^{\prime}$, we deduce that $W^{\prime}$ is concurrent with both $L^{\prime}$ and $L^{\prime \theta}$ (and note that the latter is equal to $\operatorname{proj}_{v_{i}} W^{\prime}$ ). Since $\theta^{\prime}$ now maps $L^{\prime \theta}$ back onto $L^{\prime}$, the line $Z^{\prime}:=\operatorname{proj}_{z} L^{\prime}$ is concurrent with both $\operatorname{proj}_{v_{1}} W^{\prime}$ and $\operatorname{proj}_{v_{i}} W^{\prime}$ and incident with $z$.

The lemma is proved.

If all lines of an HPMGQ satisfy Property $(\mathrm{H})$, then we can say more.

Lemma 5.7. If all lines of the thick HPMGQ $\mathcal{S}$ satisfy Property $(\mathrm{H})$ (and note that this is particularly the case if all lines of $\mathcal{S}$ are regular), then either $\mathcal{S}$ is dual classical, or the size of each span of nonconcurrent lines is equal to 2.

Proof. If there is a span of nonconcurrent lines with size at least 3, then by 5.6 .2 of FGQ, either $\mathcal{S}$ is isomorphic to the dual of $H(4, t)$, or all lines of $\mathcal{S}$ are regular. In the latter case, all lines are axes of symmetry by Theorem 3.7 of [27]. Consequently $\mathcal{S}$ is half Moufang and hence dual classical. 
The connection between HPMGQ's and GQ's having an elation point is given by the following observation.

Lemma 5.8. Let $\mathcal{S}$ be a thick HPMGQ of order $(s, t)$ with $s \leq t$, so that each span of noncollinear points has size 2 . Then each point $x$ is an elation point with corresponding elation group $H(x)$. Moreover, $H(x)$ is the complete set of elations of $\mathcal{S}$ about $x$.

Proof. Immediate from 8.2.4 (v) of FGQ and the fact that the automorphism group of $\mathcal{S}$ acts transitively on the points of $\mathcal{S}$.

These results have, for our situation, the following interesting consequence.

Corollary 5.9. Let $\mathcal{S}$ be a thick HPMGQ of order $(s, t)$ with $s \leq t$. Then $\mathcal{S}$ is classical.

Proof. According to Proposition 5.4, either $\mathcal{S}$ is classical, or each span of noncollinear points has size 2. In the latter case Lemma 5.8 and Lemma 5.6 imply that each line of $\mathcal{S}$ satisfies Property (H). Now Lemma 5.7 says that, if $\mathcal{S}$ is not classical, then the size of each span of nonconcurrent lines is equal to 2. Proposition 5.5 leads to the contradiction $s>t$.

The corollary is proved.

Henceforth we may assume that $s>t$. Roughly speaking, the idea of the rest of the proof is as follows. If there are no subquadrangles of order $\left(s^{\prime}, t\right)$ of a given HPMGQ $\mathcal{S}$ of order $(s, t)$, then in many cases it will be possible to prove that, for each point $x$, the group $H(x)$ is an elation group, and we may apply the results of the current subsection. We now investigate what we can say if there are such subquadrangles.

5.3. Observations on subquadrangles. Before considering the general case of subGQ's of order $\left(s^{\prime}, t\right)$ of HPMGQ's of order $(s, t)$, we prove a lemma with more restrictive parameters but in a slightly more general context, which we will need later on when dealing with the half 2-Moufang case.

Lemma 5.10. Let $\mathcal{S}$ be a half 2-Moufang generalized quadrangle of order $\left(q^{2}, q\right)$, $q>1$ (such that all points are centers of transitivity). If $\mathcal{S}$ admits a subquadrangle of order $(q, q)$ isomorphic to $W(q)$, then $\mathcal{S}$ is isomorphic to $H\left(3, q^{2}\right)$.

Proof. First we note that every pair of noncollinear points of $\mathcal{S}$ is contained in a subquadrangle isomorphic to $W(q)$. This easily implies that every such pair of points is regular, and hence that every subquadrangle of $\mathcal{S}$ of order $q$ is isomorphic to $W(q)$.

Fix some point $x \in \mathcal{S}$, and let $y$ and $z$ be noncollinear points in $x^{\perp}$. Let $H_{0}(x)$ be the subgroup of the group $H(x)$ of whorls about $x$ which fixes both $y$ and $z$. Let $p$ be the unique prime dividing $q$. Since $H_{0}(x)$ acts transitively on $\{y, z\}^{\perp} \backslash\{x\}$, there is a nontrivial Sylow $p$-subgroup $P$ of $H_{0}(x)$ of order at least $q$. Since $q$ and $q^{2}-1$ are relatively prime, the group $P$ fixes some point $u$ on the line $x y$ different from $x$ and $y$. Since $P$ also fixes the set $\{y, z\}^{\perp \perp}$ pointwise, it fixes all points of $\left\{u, u^{\prime}\right\}^{\perp \perp}$, with $u^{\prime} \in\{y, z\}^{\perp \perp} \backslash\{y\}$. It follows easily that $P$ fixes at least $q+1$ points incident with every line through $x$. By [29], either the fixed points of $P$ are contained in a unique subquadrangle $\mathcal{S}^{\prime}$ of order $q$ (isomorphic to $W(q)$ ), or all 
elements of $P$ are symmetries about $x$, in which case $\mathcal{S}$ is half Moufang, and hence classical and isomorphic to $H\left(3, q^{2}\right)$.

So we may assume that $P$ acts as a group of symmetries about $x$ in the subGQ $\mathcal{S}^{\prime} \cong W(q)$. Suppose by way of contradiction that $P$ does not act transitively on the set $\{y, z\}^{\perp} \backslash\{x\}$. Since $P$ has at least order $q$, this implies that some nontrivial $\theta \in P$ fixes some point $v \in\{y, z\}^{\perp} \backslash\{x\}$. Since $\theta$ induces a symmetry about $x$ in $\mathcal{S}^{\prime}$, it fixes $\mathcal{S}^{\prime}$ pointwise. But then $\theta$ is an involution (by, e.g., [29]). This implies $p=2$. Also, $\theta$ fixes $\{y, z\}^{\perp}$ pointwise, the group $\langle\theta\rangle$ is normal in $P$ (as it consists of all elements of $P$ fixing $\{y, z\}^{\perp} \backslash\{x\}$ pointwise) and the order of $P$ is equal to $2 q$ (since the order of the quotient group $P /\langle\theta\rangle$ is divisible by $q$ ). Consequently $P$ does act transitively on $\{y, z\}^{\perp} \backslash\{x\}$, a contradiction.

So $P$ acts transitively on $\{y, z\}^{\perp} \backslash\{x\}$. Since every element of $P$ fixes every point of $\mathcal{S}^{\prime}$ in $x^{\perp}$, we see that we induce the full little projective group in $\mathcal{S}^{\prime}$. By the dual of 12.10 .8 of [32] (see also Chapter 12 of [33]), the result follows.

Proposition 5.11. Suppose that $\mathcal{S}=(\mathcal{P}, \mathcal{L}, \mathrm{I})$ is a thick HPMGQ of order $(s, t)$ and let $\mathcal{S}^{\prime}=\left(\mathcal{P}^{\prime}, \mathcal{L}^{\prime}, \mathrm{I}^{\prime}\right)$ be a thick subGQ of $\mathcal{S}$ of order $\left(s^{\prime}, t\right)$. Then $\mathcal{S}^{\prime}$ is also an $H P M G Q$ (with respect to the induced group), and hence classical by Corollary 5.9.

Proof. Let $(x, L, y)$ be an arbitrary panel of $\mathcal{S}^{\prime}$ and regard it as a panel of $\mathcal{S}$. Let $V \mathrm{I} x$ and $W \mathrm{I} y$ be so that $V \neq L \neq W$, and let $M$ and $M^{\prime}$ be distinct lines, $M \neq L \neq M^{\prime}$, so that $M, M^{\prime} \in\{V, W\}^{\perp} \cap \mathcal{L}^{\prime}$. Let $\theta$ be the element of $H(x, y)$ that maps $M$ onto $M^{\prime}$. It is clear that, if for each such $(x, L, y), V, W, M, M^{\prime}$, we have that $\theta$ stabilizes $\mathcal{S}^{\prime}$ (globally), then $\mathcal{S}^{\prime}$ is an HPMGQ with respect to the stabilizer $H^{\prime}$ in $H$ of $\mathcal{S}^{\prime}$. Suppose that $\theta$ does not fix $\mathcal{S}^{\prime}$. Then by Theorem 2.1, and 2.3.1 of FGQ, $\mathcal{S}^{\prime} \cap\left(\mathcal{S}^{\prime}\right)^{\theta}$ is a subGQ of $\mathcal{S}$ of order $(1, t)$; moreover, $s^{\prime}=t$ and $s=t^{2}$. Hence $\mathcal{S}$ has a regular pair of points. Since $H$ acts transitively on the set of pairs of noncollinear points, each point of $\mathcal{S}$ is regular. This implies that each point of $\mathcal{S}^{\prime}$ is regular and so $\mathcal{S}^{\prime}$ is isomorphic to $W(t)$ by 5.2.1 of FGQ.

The proposition now follows from Lemma 5.10.

\section{Groups with a split BN-Pair of Rank 1}

We now introduce some notation that we will keep until Section 7.2 (included), unless explicitly stated otherwise.

Standing Hypotheses. From now on, $\mathcal{S}$ is a thick HPMGQ of order $(s, t), s>t$, and $V$ and $W$ are nonconcurrent lines of $\mathcal{S}$. We put

$$
\Xi=\{V, W\}^{\perp}=\left\{L_{0}, L_{1}, \ldots, L_{s}\right\},
$$

and set $v_{i}=L_{i} \cap V$, respectively $w_{i}=L_{i} \cap W$, for $i=0,1, \ldots, s$. We define $G=\left\langle H\left(v_{j}, w_{j}\right) \| j \in\{0,1, \ldots, s\}\right\rangle$. Then $(G, \Xi)$ is a (not necessarily faithful) permutation group. We denote by $N$ the kernel of the action of $G$ on $\Xi$.

Lemma 6.1. The permutation group $(G, \Xi)$ satisfies the following two properties:

(BN1) $G$ acts 2-transitively on $\Xi$, and $|\Xi|>2$;

(BN2) for every $L_{i} \in \Xi$, the stabilizer of $L_{i}$ in $G$ has a normal subgroup $H\left(v_{i}, w_{i}\right)$ which acts regularly on $\Xi \backslash\left\{L_{i}\right\}$.

Proof. This follows immediately from the definition of an HPMGQ. 
The previous lemma means that $(G, \Xi)$ is a finite group with a split BN-pair of rank 1 . We now invoke the classification of such groups (which does not depend on the classification of finite simple groups) and obtain:

Proposition 6.2. The permutation group $(G / N, \Xi)$ is either a sharply 2-transitive group, or one of the groups

(a) $\mathbf{P S L}_{2}(s), s \geq 4$,

(b) $\mathbf{R}(\sqrt[3]{s})$, a (Ree) group of Type ${ }^{2} \mathbf{G}_{2}$ in characteristic 3 ,

(c) $\mathbf{S z}(\sqrt{s}), s>4$, a (Suzuki) group of Type ${ }^{2} \mathbf{B}_{2} ; \sqrt{s}$ is then an odd exponent of 2 , or

(d) $\mathbf{P S U}_{3}\left(\sqrt[3]{s^{2}}\right)$, with $s>4$,

all acting on their natural permutation module of degree $s+1$.

Proof. This follows immediately from Lemma 6.1 and the classification of finite split BN-pairs of rank 1 by Shult [19] and Hering, Kantor and Seitz [8].

Each of the groups under $(a),(b),(c)$ and $(d)$ is nonabelian simple, except for $\mathbf{R}(3) \cong \mathbf{P} \Gamma \mathbf{L}_{2}(8)$.

We will also denote the group $H\left(v_{i}, w_{i}\right)$ by $G\left(L_{i}\right)$ and call it a root group of $(G / N, \Xi)$, or of $G / N$.

Proposition 6.2 allows us to divide the rest of the proof into different subcases according to the isomorphism class of $G / N$. We will first treat the sharply 2transitive case, and then handle the other cases (to which we will refer as "the simple case", although not all groups are simple; see above).

Remember that we use our standing hypotheses.

\section{Proof of Theorem A}

7.1. The sharply 2-transitive case. We will prove a slightly more general result, with an eye on the proof below of Theorems B and C.

Before proceeding, however, and for the convenience of the reader, we recall the following well known result, which we will use a second time later on.

Theorem 7.1 (9.4.1 of FGQ). Let $\mathcal{S}$ be a GQ of order $(s, t), s \neq 1 \neq t$. Suppose that $\Omega$ and $\Delta$ are disjoint sets of points of $\mathcal{S}$. Suppose that $K$ is a group of collineations of $\mathcal{S}$ which acts on $\Omega$, but not transitively. Suppose the following conditions are satisfied:

(1) $|\Delta|>2$;

(2) $\left|K_{y}\right|$ is independent of $y$ for $y \in \Omega$;

(3) each element of $\Omega$ is collinear with a constant number of points of $\Delta$;

(4) if $x$ and $y$ are points of $\Delta$, then there is a sequence of points $x=z_{1}, z_{2}, \ldots, z_{n}$ $=y$ so that $z_{1}, z_{2}, \ldots, z_{n} \in \Delta$, and $z_{i} \nsim z_{i+1}$ for $i=1,2, \ldots, n-1$;

(5) if $v$ is a point of $\Omega$ and $v^{\prime}$ is a point of $\Delta$ for which $v \sim v^{\prime}$, then each point of $v v^{\prime}$ which is different from $v^{\prime}$ is a point of $v^{K}$.

Then $|\Delta| \leq t+1+\bar{b}$, where $\bar{b}$ is the average number of points of $\Delta$ different from $d$ which are collinear with a given point $d \in \Delta$.

We now abandon for once our Standing Hypotheses in the first part of the next proposition.

Proposition 7.2. Let $\mathcal{S}$ be a finite thick $S E G Q$ of order $(s, t)$ and suppose that the set of elations about some point is an elation group. Let $V, W$ be two opposite 
lines in $\mathcal{S}$. Suppose that the group $E$ generated by the elations about the points of $V$ which fix $W$, acts on the points of $V$ as a sharply 2-transitive group. Then either $s \leq t$ or $\mathcal{S}$ is classical or dual classical.

In particular, under the standing hypotheses, and assuming that $(G / N, \Xi)$ is a sharply 2-transitive permutation group, the HPMGQ $\mathcal{S}$ is classical.

Proof. We can keep the notation $\Xi$ and related objects, with respect to $L, M$, as in our Standing Hypotheses. Moreover, we denote by $\Gamma(\Xi)$ the set of points on the lines of $\Xi$, and by $\Omega$ the set of points of $\mathcal{S}$ which are not on a line of $\Xi$ (so not contained in $\Gamma(\Xi)$ ). Also, define $\Delta$ as the set of points which are incident with $V$ or $W$. We assume throughout $s>t$ and show that $\mathcal{S}$ is classical.

Suppose that $G$ does not act transitively on $\Omega$. We claim that $G_{y}$ is trivial for all $y \in \Omega$. Indeed, if some nontrivial element $\theta$ of $G$ fixes $y$, then $\theta$ fixes $\operatorname{proj}_{V} y$ and $\operatorname{proj}_{W} y$ (which are not collinear!). It follows that $\theta$ fixes two elements of $\Xi$ and so $\theta \in N$. This implies that $\theta$ fixes a subGQ of order $\left(s, t^{\prime}\right), t^{\prime}>1$, contradicting $s>t$ and Theorem 2.1. We now observe that all conditions of Theorem 7.1 above are satisfied, and with $\bar{b}=s+1$, we obtain $2(s+1) \leq t+1+(s+1)$, and hence $s \leq t$, a contradiction. So $G$ acts transitively on $\Omega$. Hence the subgroup $H_{0} \leq G$ stabilizing $X=\left\{v_{0}, w_{1}\right\}^{\perp} \backslash\left\{v_{1}, w_{0}\right\}$ acts transitively on $X$, but fixes the lines $V$ and $W$ pointwise. Since $V$ and $W$ are essentially arbitrary in $\mathcal{S}$ (since $A u t(\mathcal{S})$ contains a BN-pair, it acts transitively on the ordered - ordinary - 4-gons of $\mathcal{S}$ ), the main result of $[21,22]$ implies that $\mathcal{S}$ is classical.

The second part of the proposition now follows from Corollary 5.9.

Note that, if $s>t$, the only classical quadrangles satisfying the assumption of the previous proposition have $s \in\{2,3\}$, since the 2-transitive permutation groups in question of the classical quadrangles are $\mathbf{P S L}_{2}(s)$ with their natural action.

7.2. The simple case. We use the same notation as in the previous section. In particular, $\mathcal{S}$ is an HPMGQ of order $(s, t), s>t>1$, with respect to the group $H$, and $G$ is the group generated by all $H\left(v_{i}, w_{i}\right), 0 \leq i \leq s$. The kernel of the action of $G$ on the set of lines $\Xi=\{V, W\}^{\perp}$ is denoted by $N$. By Section 7.1, we can assume that $G / N$ does not act sharply 2-transitively on $\Xi$. In particular, $s$ is a prime power. We denote $G / N$ by $K$.

We distinguish two different cases.

Proposition 7.3. If every point of $\mathcal{S}$ is regular, then $\mathcal{S}$ is classical.

Proof. First we assume that there is a subquadrangle $\mathcal{S}^{\prime}$ of order $\left(s^{\prime}, t\right)$, with $1<s^{\prime}<s$. Then by Theorem 2.1, $s=t^{2}$ and $s^{\prime}=t$ (as $t \leq s^{\prime}$, since $\mathcal{S}^{\prime}$ has regular points). Also, $\mathcal{S}^{\prime}$ is classical and isomorphic to $W(t)$ by 5.2 .1 of FGQ. By Lemma $5.10, \mathcal{S}$ is classical.

Now suppose that $\mathcal{S}$ does not admit any subquadrangle of order $\left(s^{\prime}, t\right)$, with $1<s^{\prime}<s$. The group $H\left(v_{0}\right)$ of whorls about $v_{0}$ generated by all groups of type $H\left(v_{0}, x\right)$, with $x \sim v_{0}, x \neq v_{0}$, acts transitively on the set of points of $\mathcal{S}$ not collinear with $v_{0}$. If an element of $H\left(v_{0}\right)$ fixes at least two distinct nontrivial traces in $v_{0}^{\perp}$, then by [29], either it fixes a subquadrangle of order $(t, t)$ - impossible by our assumption - or it fixes $v_{0}^{\perp}$ pointwise. Hence the action of $H\left(v_{0}\right)$ on the set of nontrivial traces in $v_{0}^{\perp}$ has a Frobenius kernel and so we obtain a group $F$, which is a normal subgroup of $H\left(v_{0}\right)$, containing the normal subgroup $N^{\prime} \unlhd H\left(v_{0}\right)$ which fixes $v_{0}^{\perp}$ pointwise and, modulo $N^{\prime}$, acting regularly on the set of nontrivial traces 
in $v_{0}^{\perp}$ (clearly, $H\left(v_{0}\right)$ acts transitively on those traces). So $F$ is defined such that $F / N^{\prime}$ is precisely the Frobenius kernel of $H\left(v_{0}\right) / N^{\prime}$ in its action on the nontrivial traces in $v_{0}^{\perp}$. It follows that $F$ acts semi-regularly on the set of points opposite $v_{0}$. But, by the definition of Frobenius kernel, $H\left(v_{0}, x\right) N^{\prime} / N^{\prime} \leq F / N^{\prime}$, so that $H\left(v_{0}, x\right) \leq F$ for all points $x$ collinear with $v_{0}$. Hence $F=H\left(v_{0}\right)$ acts sharply transitively on the set of points opposite $v_{0}$. Since the elements of $F$ fixing at least one nontrivial trace in $v_{0}^{\perp}$ are symmetries about $v_{0}$, we deduce that $F$ contains $t$ symmetries about $v_{0}$ (in fact, $\left|N^{\prime}\right|=t$ ). So $v_{0}$, and then each point of $\mathcal{S}$, is a center of symmetry, $\mathcal{S}$ is half Moufang and the proposition follows.

Proposition 7.4. If the span of every pair of noncollinear points has size 2 , then $\mathcal{S}$ is classical or dual classical.

Proof. First suppose that $\mathcal{S}$ does not admit any subquadrangle of order $\left(s^{\prime}, t\right)$, with $1<s^{\prime}<s$. This immediately implies that, with the notation of the previous proof, and using 8.1.1 of FGQ, the group $H\left(v_{0}\right)$ (and also the full group of whorls about $v_{0}$ ) either acts as a proper Frobenius group on the set of points of $\mathcal{S}$ not collinear with $v_{0}$, or it acts regularly on that set of points. If $H\left(v_{0}\right)$ does not act regularly on the points noncollinear with $v_{0}$, then by the very definition of the Frobenius kernel, all groups $H\left(v_{0}, x\right)$, with $x \neq v_{0}$ collinear with $v_{0}$, belong to the Frobenius kernel of $H\left(v_{0}\right)$. Hence $H\left(v_{0}\right)$, which is generated by those groups $H\left(v_{0}, x\right)$, coincides with its Frobenius kernel, a contradiction.

If the full group of whorls about $v_{0}$ does not coincide with $H\left(v_{0}\right)$, then by Hauptsatz 8.7 of [12], the Frobenius kernel $H\left(v_{0}\right)$, which is an elation group for the elation point $v_{0}$, is nilpotent, and hence has a nontrivial center. But then some pair of nonconcurrent lines must have a span of size at least 3 by Proposition 5.5. Consequently $\mathcal{S}$ is classical or dual classical by Lemmas 5.6 and 5.7.

So we may assume that $H\left(v_{0}\right)$ is the full group of all whorls about $v_{0}$, and it does act regularly on the set of points noncollinear with $v_{0}$. We refer to this property (which holds for all points of $\mathcal{S}$ ) as $(*)$.

Suppose $z=w_{j}$ is an arbitrary point on $W$ not collinear with $v_{0}$ (so $j \neq 0$ ), and put $\left\{v_{0}, z\right\}^{\perp}=\left\{z_{0}, z_{1}, \ldots, z_{t}\right\}$. Let $G_{i}$ be the full group of elations about $z_{i}, i=0,1, \ldots, t$, and suppose $G_{i}^{\prime}$ is the subgroup of $G_{i}$ fixing $v_{0}$ and $z$. Then Property $\left(^{*}\right)$ for the $z_{i}$ 's implies that $\left(\left\langle G_{i}^{\prime} \| i \in\{0,1, \ldots, t\}\right\rangle,\left\{v_{0}, z\right\}^{\perp}\right)$ is a group $K$ with a split BN-pair of rank 1 (the root groups are the $G_{i}^{\prime}$ 's). Suppose first that $K$ does not act sharply 2-transitively on $\left\{v_{0}, z\right\}^{\perp}$. Then there is a prime $p^{\prime}$ and a natural number $h^{\prime}$ with $t=p^{h^{\prime}}$. Put $s=p^{h}$, with $p$ prime and $h$ a positive integer (recall that we are in the simple case !). The group $H\left(v_{0}, w_{0}\right)$ fixes some third point $u_{0}$ on the line $L_{0}=v_{0} w_{0}$, and if $p^{\prime} \neq p$, then clearly it also fixes some second line $U_{0}$ through $u_{0}, U_{0} \neq L_{0}$. Hence $U_{0}$ is contained in $\left\{V, W^{\prime}\right\}^{\perp \perp}$ for an appropriate line $W^{\prime}$ through $w_{0}$. Lemmas 5.6 and 5.7 imply that $\mathcal{S}$ is classical or dual classical. So we may assume $p=p^{\prime}$ and $H\left(v_{0}\right)$ is a $p$-group, hence has a nontrivial center. As in the previous paragraph, we conclude that $\mathcal{S}$ is classical or dual classical. Note that these arguments apply as soon as $t$ is a prime power.

Consequently we may assume that $K$ acts sharply 2-transitively on $\left\{v_{0}, z\right\}^{\perp}$. Clearly, the group $G_{L_{0}, L_{j}}$ acts as an automorphism group of $K$. Now, no nontrivial $\sigma \in G_{L_{0}, L_{j}}$ fixes $\left\{v_{0}, z\right\}^{\perp}$ pointwise (as otherwise $\sigma$ would be a nontrivial whorl about both $v_{0}$ and $z$, clearly a contradiction to Property $\left(^{*}\right)$ applied to $v_{0}$ and $z$ ), so $G_{L_{0}, L_{j}}$ acts as a faithful automorphism group on $K$. Note also that $G_{L_{0}, L_{j}}$ fixes at least two points of $\left\{v_{0}, z\right\}^{\perp}$ (namely, $w_{0}$ and $v_{j}$ ). 
First assume that $K$ is not solvable. Then $t+1 \in\left\{11^{2}, 29^{2}, 59^{2}\right\}$ (see 20.2 of [14]). Identifying $\left\{v_{0}, z\right\}^{\perp}$ with the 2-dimensional vector space over the field with $\sqrt{t+1}$ elements, we see that a nontrivial element $\sigma$ of $G_{L_{0}, L_{j}}$ induces in the stabilizer of the zero vector an element of $\mathbf{G L}_{2}(\sqrt{t+1})$ that normalizes the (maximal) subgroup $\mathbf{S L}_{2}(5)$; hence $\sigma$ is a scalar. But a scalar can only fix a nonzero vector if it is the identity. Consequently $G_{L_{0}, L_{j}}$ must be trivial, a contradiction.

Hence we may assume that $K$ is solvable. Denote by $T$ the permutation group acting on $\left\{v_{0}, z\right\}^{\perp}$ and generated by $K$ and $G_{L_{0}, L_{j}}$. Then either $T$ is a subgroup of $\mathbf{A} \mathbf{L}_{2}(t+1)$, or $t+1 \in\left\{3^{2}, 3^{4}, 5^{2}, 7^{2}, 11^{2}, 23^{2}\right\}$ (see 19.9 of [14]). Putting $t+1=p^{h^{\prime}}$, with $p^{\prime}$ prime and $h^{\prime}$ a positive integer, we see that in the first case $\left|G_{L_{0}, L_{j}}\right| \leq h^{\prime}$ (as $\left.\left|\mathbf{A} \boldsymbol{\Gamma} \mathbf{L}_{2}(t+1)\right|=(t+1) t h^{\prime}\right)$. An easy inspection reveals that this is only possible if $s=27, t+1=16$ and $G / N$ is the Ree group $\mathbf{P} \Gamma \mathbf{L}_{2}(8)$ of degree 28 . In this case $G_{L_{0}, L_{j}}$ contains a unique involution $\theta$, which fixes a subquadrangle $\mathcal{S}^{\prime}$ of order $(3,3)$ pointwise. It is easy to see that the full little projective group of $\mathcal{S}^{\prime}$ is induced by the collineation group of $\mathcal{S}$. Hence this implies that there is an involutory automorphism of $\mathbf{P} \boldsymbol{\Gamma} \mathbf{L}_{2}$ (8) fixing in the corresponding Ree unital two points $a, b$ and interchanging the two other points on the block though $a$ and $b$, a contradiction (all involutions in $\mathbf{P} \boldsymbol{\Gamma} \mathbf{L}_{2}(8)$ are conjugate and they fix exactly 4 points on a block). Hence we have $t+1 \in\left\{3^{2}, 3^{4}, 5^{2}, 7^{2}, 11^{2}, 23^{2}\right\}$. If $t+1 \in\left\{5^{2}, 7^{2}, 11^{2}, 23^{2}\right\}$, then a similar argument as above (now with $\mathbf{S L}_{2}(3)$ instead of $\mathbf{S L}_{2}(5)$ ) shows that $G_{L_{0}, L_{j}}$ must be trivial, a contradiction. So there remains to consider the cases $t+1 \in\left\{3^{2}, 3^{4}\right\}$. If $t+1=3^{2}$, then $t=2^{3}$ and so $t$ is a prime power. We remarked before that this leads to $\mathcal{S}$ being classical or dual classical. So $t+1=3^{4}$. If $s$ is not divisible by 2 or 5 , then we can argue as in the case where $t$ is a prime power to conclude that $\mathcal{S}$ is classical or dual classical. Hence $s \in\left\{2^{7}, 2^{8}, \ldots, 2^{12}, 5^{3}, 5^{4}, 5^{5}\right\}$, because $t<s \leq t^{2}$. But all of these possibilities violate the fact that $\frac{s t(1+s t)}{s+t}$ must be an integer.

We conclude that $\mathcal{S}$ admits a subGQ $\mathcal{S}^{\prime}$ of order $\left(s^{\prime}, t\right)$, with $1<s^{\prime}<s$, which is half pseudo Moufang by Proposition 5.11. By Corollary 5.9, $\mathcal{S}^{\prime}$ is classical (as $\left.s^{\prime} \leq t\right)$. So we have the following three possibilities:

(a) $\mathcal{S}^{\prime} \cong \mathcal{Q}(4, t)$

(b) $\mathcal{S}^{\prime} \cong H\left(4, \sqrt[3]{t^{2}}\right)$;

(c) $\mathcal{S}^{\prime} \cong \mathcal{Q}(5, \sqrt{t})$.

In Case (a), any line not belonging to $\mathcal{S}^{\prime}$ subtends some spread of $\mathcal{S}^{\prime}$, implying by 3.4.1 of FGQ that $t$ is even; but then each span of noncollinear points has size $t$, a contradiction. Case (b) is not possible as each span of noncollinear points of $H\left(4, \sqrt[3]{t^{2}}\right)$ has size $\sqrt[3]{t}+1$; see 3.3.1 of FGQ. Finally, in Case (c), putting $q^{2}=t$, the stabilizer of $L_{0}, L_{1}$ in the group $G / N$ must have orbits whose union has size $q+1$ (otherwise $\mathcal{S}^{\prime}$, which can be assumed to contain $V, W, L_{0}, L_{1}$, has an image containing $L_{0}, L_{1}, V, W$ and distinct from $\mathcal{S}^{\prime}$, a contradiction, as this would imply that $\mathcal{S}$ has regular pairs of points). Also, clearly $q$ divides $s=\left|H\left(v_{0}, w_{0}\right)\right|$ (by considering the number of $\mathcal{Q}(5, q)$ subGQ's through $\left.v_{0}, v_{0} w_{0}, w_{0}\right)$. By inspection, this immediately implies that $G / N$ is isomorphic to either $\mathbf{P S U}_{3}\left(q^{2}\right)$ or $\mathbf{R}(q)$. Hence $(s, t)=\left(q^{3}, q^{2}\right)$ and $\mathcal{S}$ is dual to $H(4, t)$ by the fact that $H$ acts transitively on the pairs of noncollinear points and by applying the dual of Appendix B of [24].

This completes the proof of the proposition.

The proof of Theorem A is thus complete. 


\section{Classification of (finite) half 2-Moufang GENERALIZED QUADRANGLES}

We prove Theorem B.

Let $\mathcal{S}=(\mathcal{P}, \mathcal{L}, \mathrm{I})$ be a thick generalized quadrangle, each point of which is a center of transitivity. Let $V$ and $W$ be nonconcurrent lines of $\mathcal{S}$. Put $\Xi=$ $\{V, W\}^{\perp}=\left\{L_{0}, L_{1}, \ldots, L_{s}\right\}$, and set $v_{i}=L_{i} \cap V$ and $w_{i}=L_{i} \cap W$, for $i=0,1, \ldots, s$. We will sometimes treat $w_{0}$ as an arbitrary point on $L_{0}$ distinct from $v_{0}$, and $W$ as an arbitrary line through $w_{0}$ distinct from $L_{0}$.

We denote by $H$ an automorphism group of $\mathcal{S}$ containing all the whorls about points.

By Proposition 5.4, and the fact that every quadrangle of order $(s, s)$ all points of which are regular is isomorphic to $W(s)$, we have, similarly as in Proposition 5.4, that either $\mathcal{S}$ is classical, or the span of every pair of points has size 2 , or $t<s$ and every point is regular. We now treat the two latter cases.

8.1. Every point is regular and $t<s$. Here, there are two subcases, just as in Proposition 7.3.

First suppose that there is no subquadrangle of order $\left(s^{\prime}, t\right)$, with $1<s^{\prime}<s$. Let $H\left(v_{0}\right)$ be the group of whorls about $v_{0}$; then, as in the proof of Proposition 7.3, $H\left(v_{0}\right)$ contains a subgroup $F$ containing all symmetries about $v_{0}$ - say that the group of these symmetries is $N^{\prime}$ - so that $F / N^{\prime}$ acts regularly on the set of nontrivial traces in $v_{0}^{\perp}$ (that is, $F / N^{\prime}$ is the Frobenius kernel of the Frobenius group $H\left(v_{0}\right) / N^{\prime}$ in its action on the set of nontrivial traces in $\left.v_{0}^{\perp}\right)$. Also, $F$ is a group of elations about $v_{0}$. Now let $R$ be an arbitrary line not incident with $v_{0}$, and let $p$ be an arbitrary prime dividing $s$. Let $S$ be a Sylow $p$-subgroup in $H\left(v_{0}\right)_{R}$. Then $S$ is a group of elations about $v_{0}$, as there are no subGQ's of order $\left(s^{\prime}, t\right)$, $s^{\prime}>1$, in $\mathcal{S}$. Whence $S N^{\prime} / N^{\prime}$ is a subgroup of $F / N^{\prime}$, so that $S \leq F$. As $p$ was arbitrary, we conclude that $H\left(v_{0}\right)_{R} \leq F_{R}$. As there are no thick subGQ's of order $\left(s^{\prime}, t\right)$, it follows that $F_{R}$ acts transitively, and then regularly, on the points on $R$ which are not collinear with $v_{0}$. Since $R$ is arbitrary, we can conclude that $F$ acts regularly on the points not collinear with $v_{0}$, so that $\left|N^{\prime}\right|=t$ (because $|F|=s^{2} t$ and the number of nontrivial traces in $v_{0}^{\perp}$ is equal to $s^{2}$ ). Whence $v_{0}$ is a center of symmetry. The result follows as in Proposition 7.3.

Next suppose that there is some subquadrangle $\mathcal{S}^{\prime}$ of order $\left(s^{\prime}, t\right)$, with $1<s^{\prime}<s$. As in the proof of Proposition 7.3, $\mathcal{S}^{\prime}$ is isomorphic to $W(t)$ and $s=t^{2}$. The result now follows from Lemma 5.10.

8.2. The span of every pair of noncollinear points has size 2. Again, we distinguish two cases with respect to subquadrangles.

8.2.1. First case: $\mathcal{S}$ does not admit a subGQ of order $\left(s^{\prime}, t\right), 1<s^{\prime}<s$. We start with a lemma on finite Chevalley groups of rank 1.

Lemma 8.1. Let $K$ be a group acting on $X$ and suppose that the permutation group $(K, X)$ is permutation equivalent to the action of $\mathbf{P S L} \mathbf{L}_{2}(q)(q \geq 4), \mathbf{P S U}_{3}(q)$ $(q \geq 3), \mathbf{S z}(q)$ ( $q \geq 8)$ or $\mathbf{R}(q)$ ( $q \geq 3)$, with $q$ an appropriate prime power, on the natural permutation module (respectively of size $q+1, q^{3}+1, q^{2}+1$ or $\left.q^{3}+1\right)$. Let $x \in X$ be arbitrary. Let $P$ be the unique Sylow p-subgroup of $K_{x}$ (which is also a regular - on $X \backslash\{x\}-$ normal subgroup of $\left.K_{x}\right)$. Then $\left[P, K_{x}\right]=P$. 
Proof. Choose $y \in X \backslash\{x\}$ arbitrarily. First of all, note that $\left[P, K_{x}\right] \leq P$, as $P$ is normal in $K_{x}$. It is easy to check that in all cases, there is an element $\varphi \in K_{x, y}$ fixing only $x, y$. Let $\alpha, \beta \in P$ with $\alpha \neq \beta$ and both nontrivial. Suppose $[\varphi, \alpha]=[\varphi, \beta]$; then $\varphi^{\alpha}=\varphi^{\beta}$. But $\varphi^{\alpha}$ fixes only $y^{\alpha}$ in $X \backslash\{x\}$, while $\varphi^{\beta}$ fixes only $y^{\beta}$ in $X \backslash\{x\}$, a contradiction. So $[\varphi, P]=P$.

The lemma is proved.

We now continue with the proof of Theorem B and assume that $\mathcal{S}$ does not admit a subGQ of order $\left(s^{\prime}, t\right), 1<s^{\prime}<s$. Note that this assumption is automatic whenever $s \leq t$. It is easy to see that the group $H\left(v_{0}\right)$ of whorls about $v_{0}$ is a Frobenius group on the set of points opposite $v_{0}$ (cf. 8.1.1 of FGQ). The Frobenius kernel $F\left(v_{0}\right)$ consists of the set of all elations about $v_{0}$. In particular $\mathcal{S}$ is an SEGQ.

Let $H\left(W, v_{i}\right), i=0,1, \ldots, s$, be a group of elations about $v_{i}$ which fixes $W$ and acts regularly on $W \backslash\left\{w_{i}\right\}$ (that exists since $\mathcal{S}$ is an SEGQ). In a similar fashion, we define $H\left(V, w_{i}\right)$. The group $G(V)=\left\langle H\left(W, v_{j}\right) \| j \in\{0,1, \ldots, s\}\right\rangle$ acts as a split BN-pair of rank 1 on the set $\Xi$. Similarly, the group $G(W)$, defined analogously, also acts as a split BN-pair of rank 1 on $\Xi$. Clearly $G(W)$ acts as an automorphism group on $G(V)$ and is normal in $\langle G(V), G(W)\rangle$.

Suppose now first that $G(V)$ is related to a simple group (the non sharply-2transitive case, where $G(V)$ modulo the kernel is not isomorphic to $\mathbf{R}(3))$. We first claim that the action of $G(V)$ on $\Xi$ must coincide with the action of $G(W)$ on $\Xi$. Indeed, suppose not. We denote the (faithful) actions of $G(V)$ and $G(W)$ on $\Xi$ by $G_{V}$ and $G_{W}$, respectively (and both $G_{V}$ and $G_{W}$ are simple groups). Since $H\left(W, v_{i}\right)$ and $H\left(V, w_{i}\right)$ normalize each other, for all $i=0,1, \ldots, s$, the groups $G_{V}$ and $G_{W}$ normalize each other. Hence $G_{V}, G_{W} \unlhd\left\langle G_{V}, G_{W}\right\rangle$, and consequently $G_{V} \cap G_{W} \unlhd G_{V}$, implying $\left|G_{V} \cap G_{W}\right|=1$. But then $G_{V}$ and $G_{W}$ centralize each other. This now easily leads to a contradiction. Our claim is proved.

Now let $\varphi \in G(V)_{v_{i}}$ be arbitrary, $i \in\{0,1, \ldots, s\}$, and let $u \in H\left(W, v_{i}\right)$ be arbitrary. By our previous claim, there is a unique element $u^{\prime} \in H\left(V, w_{i}\right)$ such that $u u^{\prime}$ fixes all points on both $V$ and $W$. Clearly, $u u^{\prime}$ centralizes every element of $H\left(W, v_{j}\right)$, for all $j \in\{0,1, \ldots, s\}$. This means that $u u^{\prime}$ commutes with $\varphi$. Hence, the actions of $\varphi$ and $u u^{\prime}$ on the set of lines through the point $w_{i}$ commute. Since $u^{\prime}$ has trivial action on that set, this implies that $[\varphi, u] \in H\left(W, v_{i}\right) \cap H\left(V, w_{i}\right)$. Now from Lemma 8.1 it follows that $H\left(W, v_{i}\right)=H\left(V, w_{i}\right)$ and so $\mathcal{S}$ is an HPMGQ, hence classical or dual classical.

Suppose now that $G(V)$ is related to $\mathbf{R}(3)$. As no element of $G(W)$ modulo the kernel $\left(N_{W}\right)$ acts trivially on $G(V)$ modulo the kernel $\left(N_{V}\right), G(W) / N_{W}$ is a faithful automorphism group of $G(V) / N_{V}$. So $G(W) / N_{W} \leq \operatorname{Aut}\left(G(V) / N_{V}\right)=$ $G(V) / N_{V} \cong \mathbf{R}(3)$, and hence $G(W)$ and $G(V)$ have the same action on $\Xi$. One now proceeds as in the previous paragraph.

So we may assume that the action of $G(V)$ on $\Xi$ is sharply 2-transitive. By Proposition 7.2, $s \leq t$. By [7], $s$ and $t$ are powers of the same prime $p$. If $s=p$, then by [1], $\mathcal{S}$ is classical. Now suppose that $s+1$ is a prime. Then the action of $G(V)$ on $\Xi$ must coincide with the action of $G(W)$ on $\Xi$, because $H\left(W, v_{i}\right)$ acts as an automorphism group on $G(W)$, and hence on the Frobenius kernel of $G(W)$, which is a group of prime order.

Now, if $H\left(W, v_{0}\right)=H\left(V, w_{0}\right)$, then, as before, we see that $\mathcal{S}$ is half pseudo Moufang and hence classical or dual classical. Otherwise, since $s$ and $t$ are both powers of the prime 2 , the group $H\left(W, v_{0}\right)$ fixes a line through $w_{0}$ different from 
$L_{0}$ and from $W$ (but it does not fix all lines through $w_{0}$ ). Consider any nontrivial $\theta \in H\left(W, v_{0}\right)$. If $\theta^{\prime} \in H\left(V, w_{0}\right)$ is such that $\theta \theta^{\prime}$ acts as the identity on $\Xi$, then the fixed point structure of $\theta \theta^{\prime}$ is a subquadrangle $\mathcal{S}_{\theta}^{\prime}$ of order $\left(s, t_{\theta}\right)$, with $t_{\theta}>1$. The intersection of all $\mathcal{S}_{\theta}$ 's is a subquadrangle $\mathcal{S}^{\prime}$ of order $\left(s, t^{\prime}\right)$, with $1<t^{\prime} \leq s \leq t$ (in fact, one notes that $\left.t^{\prime}=t_{\theta}\right)$, stabilized under the action of $H\left(W, v_{0}\right)$. Moreover, the action of $H\left(W, v_{0}\right)$ on $\mathcal{S}^{\prime}$ coincides with the action on $\mathcal{S}^{\prime}$ of $H\left(V, w_{0}\right)$. Hence $\mathcal{S}^{\prime}$ is an HPMGQ, and so classical or dual classical. In $\mathcal{S}^{\prime}$, the group $H\left(W, v_{0}\right)$ is a root group. But $H\left(W, v_{0}\right)$ is cyclic of order $s$, and abelian root groups are elementary abelian, implying that $s=2$, so that $\mathcal{S}$ is classical.

Hence we may assume that neither $s$ nor $s+1$ is a prime. This implies by a straightforward arithmetical argument - see e.g. 19.3 of [14] - that $s=8$. Now, there are exactly two nonisomorphic sharply 2-transitive groups of degree $9=s+1$ (see [14]). They are obtained as follows. We may identify the set being permuted with a 2 -dimensional vector space over $\mathbf{G F}(3)$, and the Frobenius kernel is the translation group. The stabilizer of the zero vector is either a cyclic group of order 8, or a quaternion group of order 8. It is easy to verify that $\mathbf{G L}_{2}(3)$ contains a unique subgroup isomorphic to a quaternion group of order 8 , and that two distinct cyclic groups of order 8 in $\mathbf{G L}_{2}(3)$ generate $\mathbf{G L}_{2}(3)$, and hence can never normalize each other. This immediately implies that $H\left(W, v_{0}\right)$ acts in the same way on $\Xi$ as $H\left(V, w_{0}\right)$ (since clearly $H\left(V, w_{0}\right)$ normalizes $G(V)$, hence preserves the vector space structure, hence can be identified with a subgroup of $\mathbf{G L}_{2}(3)$, and hence normalizes $H\left(W, v_{0}\right)$; note that $H\left(W, v_{0}\right) \cong H\left(V, w_{0}\right)$ for transitivity reasons). As in the previous paragraph, we obtain a classical subGQ $\mathcal{S}^{\prime}$ with root groups isomorphic to either a cyclic group of order 8 (again a contradiction), or a quaternion group of order 8. Hence $\mathcal{S}^{\prime}$ is isomorphic to the dual of $H(4,4)$. But then a point $x$ of $\mathcal{S}$ outside $\mathcal{S}^{\prime}$ subtends an ovoid in $\mathcal{S}^{\prime}$ (the set of points of $\mathcal{S}^{\prime}$ collinear with $x$ ). Brouwer (1981, unpublished) has proved though that $H(4,4)$ has no spreads. Hence this case does not occur.

8.2.2. Second case: $\mathcal{S}$ admits subGQ's of order $\left(s^{\prime}, t\right), 1<s^{\prime}<s$. Without loss of generality we may assume that a subGQ $\mathcal{S}^{\prime}$ of order $\left(s^{\prime}, t\right)$ contains $V, W, v_{0}, v_{1}$ (and hence $\left.w_{0}, w_{1}\right)$. Every whorl about $v_{0}$ mapping $w_{1}$ onto another point of $\mathcal{S}^{\prime}$ preserves $\mathcal{S}^{\prime}$, as otherwise the intersection of $\mathcal{S}^{\prime}$ with its image is a subquadrangle of order $(1, t)$, contradicting the fact that the span of every pair of noncollinear points has size 2 (as well in $\mathcal{S}^{\prime}$ as in $\mathcal{S}$ ). Hence every point of $\mathcal{S}^{\prime}$ is a center of transitivity, and by the first part of the proof (and the fact that, as $s^{\prime} \leq t, \mathcal{S}^{\prime}$ cannot have a subquadrangle of order $\left(s^{\prime \prime}, t\right)$ with $\left.1<s^{\prime \prime}<s^{\prime}\right) \mathcal{S}^{\prime}$ is a dual classical quadrangle. By inspection $\mathcal{S}^{\prime}$ is either isomorphic to $\mathcal{Q}\left(4, s^{\prime}\right)$ with $s^{\prime}$ odd (a contradiction since any line of $\mathcal{S}$ outside $\mathcal{S}^{\prime}$ subtends a spread of $\mathcal{S}^{\prime}$ and $\mathcal{Q}\left(4, s^{\prime}\right)$ has no spreads by 3.4.1(i) of FGQ), or $\mathcal{S}^{\prime}$ is isomorphic to $\mathcal{Q}(5, \sqrt{t})$, with $s^{\prime}=\sqrt{t}$. We can put $t=q^{2}$, with $q$ a prime power.

Let $H^{\prime}$ be the stabilizer of $\mathcal{S}^{\prime}$ in $H$. Then the restriction of $H^{\prime}$ to $\mathcal{S}^{\prime}$ contains the little projective group $\mathbf{P S U}(4, q)$ of $\mathcal{S}^{\prime}$.

The rest of the proof is subdivided as follows. First we derive a divisibility condition in the general case, and then we distinguish between $q$ odd and $q$ even. In both cases we use properties of involutions in $H^{\prime}$ to show that $\mathcal{S}$ is dual to $H\left(4, q^{2}\right)$. In the course of the proof, we will therefore have to establish some lemmas about involutions acting on $\mathcal{Q}(5, q)$. 
A divisibility condition. We claim that $s-q$ is divisible by $q(q-1)$. Indeed, from a previous discussion it follows that every pair of opposite points of $\mathcal{S}$ is contained in exactly one subquadrangle of order $\left(q, q^{2}\right)$. Let $n$ be the number of such subquadrangles containing some fixed (but arbitrary) point of $\mathcal{S}$. Counting in two ways the number of triples $\left(\mathcal{S}^{\prime \prime}, x, y\right)$, with $\mathcal{S}^{\prime \prime}$ a subquadrangle of order $\left(q, q^{2}\right)$, and $x, y$ a pair of opposite points of $\mathcal{S}^{\prime \prime}$, we obtain $(1+s)\left(1+s q^{2}\right) \times n \times q^{4}=$ $(1+s)\left(1+s q^{2}\right) \times s^{2} q^{2} \times 1$, from which it follows that $n=s^{2} / q^{2}$. Hence $q$ divides $s$.

Using the criterion given in the proof of Theorem 8.3.3 of [43], it is easy to see that $H^{\prime}$ contains an element $h$ (a so-called generalized homology, living in a torus of $\mathbf{S U}_{4}\left(q^{2}\right)$ ) which is a whorl about two opposite points, say $v_{0}$ and $w_{1}$, of $\mathcal{S}^{\prime}$, and whose restriction to $\mathcal{S}^{\prime}$ is of order $q-1$. Hence $h$ has order $(q-1) k$, for some positive integer $k$. Suppose some nontrivial element $h^{\prime}$ of $\langle h\rangle$ fixes a point $x$ on the line $V$, outside $\mathcal{S}^{\prime}$. Then $h^{\prime}$ fixes pointwise a subquadrangle of some order $\left(s^{\prime}, q^{2}\right)$, which must necessarily coincide with $\mathcal{S}^{\prime}$ (because the order of the intersection must be $\left.\left(q, q^{2}\right)\right)$, a contradiction. Hence $\langle h\rangle$ acts semi-regularly on the point set of $V$ outside $\mathcal{S}^{\prime}$, which has size $s-q$. It follows that $(q-1) k$, and hence $q-1$, divides $s-q$.

We conclude that $q(q-1)$ divides $s-q$, as $q$ and $q-1$ are relatively prime.

Some properties of fixed point free involutions of $\mathcal{Q}(5, q), q$ odd. We will need some specific properties of involutions of $\mathcal{Q}(5, q)$, which we now establish. The proofs use standard calculations, and we shall content ourselves by mentioning the main steps. Throughout, we assume that $q$ is odd.

Throughout, we consider the quadric $\mathcal{Q}(5, q)$ of $\mathbf{P G}(5, q)$ with equation $X_{0} X_{1}+$ $X_{2} X_{3}=X_{4}^{2}-\not \supset X_{5}^{2}$, where $\square$ is some fixed nonsquare in the field $\mathbf{G F}(q)$. The point with coordinates $(0, \ldots, 0,1,0, \ldots, 0)$, where the 1 is in the $i$ th position, will be denoted by $e_{i-1}$ (and so $\left\{e_{0}, e_{1}, \ldots, e_{5}\right\}$ is a generating set of points for $\mathbf{P G}(5, q)$ ). A Hermitian spread of $\mathcal{Q}(5, q)$ is a set of $q^{3}+1$ mutually opposite lines of $\mathcal{Q}(5, q)$ with the property that for any two lines $L, M$ of this spread, the set $\{L, M\}^{\perp \perp}$ belongs to the spread.

Lemma 8.2. Let $\sigma$ be a fixed point free involution of $\mathcal{Q}(5, q), q$ odd. Then either $\sigma$ fixes a Hermitian spread of $\mathcal{Q}(5, q)$ elementwise (and every point is mapped onto a collinear one), or $\sigma$ fixes elementwise a set $S$ of $q^{2}+1$ lines with the property that for every two lines $L, M \in S$, one has $S \cap\{L, M\}^{\perp \perp}=\{L, M\}$, and consequently for every line $X \notin S$ of $\mathcal{Q}(5, q)$, the set $\left\{X, X^{\sigma}\right\}^{\perp \perp} \cup\left\{X, X^{\sigma}\right\}^{\perp}$ meets $S$ in exactly 2 elements (and in this case there exists a point which is mapped onto an opposite point by $\sigma$ ).

Proof. Note first that $\sigma$ must arise from an involution of $\mathbf{P G}(5, q)$; see for instance Proposition 4.6.3 of [43]. Clearly $\sigma$ does not fix all lines of $\mathcal{Q}(5, q)$, hence we may without loss of generality assume that $\sigma$ does not fix $e_{0} e_{2}$. The image of this line cannot be concurrent with it (as the intersection would be a fixed point), hence we may without loss of generality assume that $\left(e_{0} e_{2}\right)^{\sigma}=e_{1} e_{3}$. Hence $\sigma$ stabilizes the two sets of $q+1$ lines determined by the hyperbolic quadric $\mathcal{Q}^{+}(3, q)$ obtained from $\mathcal{Q}(5, q)$ by intersecting with the space $\mathbf{P G}(3, q)$ having equations $X_{4}=X_{5}=0$. As these sets behave as projective lines with respect to their stabilizers, we already deduce that $\sigma$ is a linear collineation, i.e., the companion field automorphism of $\sigma$ is the identity (otherwise $\sigma$ fixes $\sqrt{q}+1$ lines of each set, and hence at least $(\sqrt{q}+1)^{2}$ points, a contradiction). We now show that there is at least one fixed line. 
Assume, by way of contradiction, that $\sigma$ does not fix any line of $\mathcal{Q}(5, q)$. The restriction of $\sigma$ to $\mathbf{P G}(3, q)$ has, without loss of generality, the following description:

$$
\sigma: \mathbf{P G}(3, q) \rightarrow \mathbf{P G}(3, q):\left(x_{0}, x_{1}, x_{2}, x_{3}\right) \mapsto\left(k \ell x_{1}, x_{0}, \ell x_{3}, k x_{2}\right),
$$

with $k, \ell \in \mathbf{G F}(q)$. Our assumption translates to $-k$ and $-\ell$ being nonsquares in $\mathbf{G F}(q)$, implying that $k \ell$ is a square, with square roots, say, $\sqrt{k \ell}$ and $-\sqrt{k \ell}$. Hence the point with coordinates $(\sqrt{k \ell}, 1,0,0)$ (which is not a point of $\mathcal{Q}(5, q)$ ) is fixed by $\sigma$, implying that $\sigma$ fixes a 4 -dimensional projective space which meets $\mathcal{Q}(5, q)$ in a nonsingular quadric isomorphic to $\mathcal{Q}(4, q)$. Without loss of generality, we may take as an equation for that 4 -space $X_{5}=0$, still with the space with equations $X_{4}=X_{5}=0$ being fixed, too. It also follows that the space with equation $X_{4}=0$ is fixed (recall that $\mathcal{Q}(5, q)$ is fixed), and so $\sigma$ can be written as follows:

$$
\begin{gathered}
\sigma: \mathbf{P G}(5, q) \rightarrow \mathbf{P G}(5, q):\left(x_{0}, x_{1}, x_{2}, x_{3}, x_{4}, x_{5}\right) \\
\mapsto\left(k \ell x_{1}, x_{0}, \ell x_{3}, k x_{2}, \sqrt{k \ell} x_{4}, \epsilon \sqrt{k \ell} x_{5}\right),
\end{gathered}
$$

where we possibly have to interchange the two notations $\sqrt{k \ell}$ and $-\sqrt{k \ell}$ for the square roots of $k \ell$, and where $\epsilon \in\{+1,-1\}$. If $\epsilon=+1$, then for every $a, b, c \in$ $\mathbf{G F}(q)$, the point with coordinates $(\sqrt{k \ell}, 1, a \ell, a \sqrt{k \ell}, b, c)$ is fixed by $\sigma$. Such a point belongs to $\mathcal{Q}(5, q)$ if and only if $\sqrt{k \ell}+a^{2} \ell \sqrt{k l}=b^{2}-\not \subset c^{2}$, which always has solutions in $a, b, c$ over $\mathbf{G F}(q)$, a contradiction. Hence $\epsilon=-1$. If $\sqrt{k \ell}$ is a square in $\mathbf{G F}(q)$, say $a^{2}=\sqrt{k \ell}$, then the point with coordinates $(\sqrt{k \ell}, 1,0,0, a, 0)$ is fixed by $\sigma$ and belongs to $\mathcal{Q}(5, q)$. Otherwise, $\sqrt{k \ell}$ may be written as $\not \subset b^{2}$, and then the point with coordinates $(-\sqrt{k \ell}, 1,0,0,0, b)$ is fixed by $\sigma$ and belongs to $\mathcal{Q}(5, q)$. Both are contradictions, and we can conclude that $\sigma$ fixes at least one line.

Since the number of lines is even, $\sigma$ has to fix at least two lines. Without loss of generality we may assume that $\sigma$ fixes the lines $e_{0} e_{3}$ and $e_{1} e_{2}$. Hence the restriction of $\sigma$ to $\mathbf{P G}(3, q)$ looks as follows:

$$
\sigma: \mathbf{P G}(3, q) \rightarrow \mathbf{P G}(3, q):\left(x_{0}, x_{1}, x_{2}, x_{3}\right) \mapsto\left(\epsilon \ell x_{3}, \epsilon x_{2}, \ell x_{1}, x_{0}\right),
$$

with $\ell \in \mathbf{G F}(q)$, and $\epsilon \in\{+1,-1\}$. Since $\sigma$ has no fixed points on $\mathcal{Q}^{+}(3, q)$, we deduce that $\epsilon \ell$ is not a square in $\mathbf{G F}(q)$. Suppose now that the restriction of $\sigma$ to $e_{4} e_{5}$ is the identity. Then $\sigma$ maps

$$
\left(x_{0}, x_{1}, x_{2}, x_{3}, x_{4}, x_{5}\right) \text { to }\left(\epsilon \ell x_{3}, \epsilon x_{2}, \ell x_{1}, x_{0}, a x_{4}, a x_{5}\right),
$$

for some $a \in \mathbf{G F}(q)^{*}$, and this is of order 2 only if $\epsilon \ell=a^{2}$, a contradiction. Hence there exist elements $a_{1}, a_{2}, a_{3} \in \mathbf{G F}(q)$ such that $\sigma$ can be represented as follows:

$$
\begin{aligned}
& \sigma: \mathbf{P G}(5, q) \rightarrow \mathbf{P G}(5, q):\left(x_{0}, x_{1}, x_{2}, x_{3}, x_{4}, x_{5}\right) \\
& \mapsto\left(\epsilon \ell x_{3}, \epsilon x_{2}, \ell x_{1}, x_{0}, a_{1} x_{4}+a_{2} x_{5}, a_{3} x_{4}-a_{1} x_{5}\right) .
\end{aligned}
$$

An elementary calculation now shows that, expressing that $\sigma$ preserves $\mathcal{Q}(5, q)$ and $\sigma$ is involutory, we have only the following two possibilities:

(1) $\epsilon=-1$.

Then $\ell=-\not a_{3}^{2}, a_{2}=\not a_{3}$ and $a_{1}=0$. It is easily checked that every point of $\mathbf{P G}(5, q)$ is conjugate to its image under $\sigma$ with respect to the bilinear form associated with $\mathcal{Q}(5, q)$. Hence every point of $\mathcal{Q}(5, q)$ is incident with exactly one fixed line. The set of fixed lines thus forms a spread. Let $L, M$ be two lines of that spread, and suppose that $x$ is a point of $\mathcal{Q}(5, q)$ incident with a line $K$ which meets both $L$ and $M$. Then $x^{\sigma}$ should be contained in $\langle L, M\rangle$, and should also be collinear 
with $x$ on $\mathcal{Q}(5, q)$. This is clearly only possible if $x x^{\sigma}$ belongs to $\{L, M\}^{\perp \perp}$. We have shown that the set of fixed lines is a Hermitian spread of $\mathcal{Q}(5, q)$.

(2) $\epsilon=1$

Here, $a_{2}=-\not \triangleright a_{3}$ and $\ell=a_{1}^{2}-\not \triangleright a_{3}^{2}$. In this case, the coordinates of the points of $\mathcal{Q}(5, q)$ which are mapped onto a collinear point of $\mathcal{Q}(5, q)$ are given by $\left(x_{0}, x_{1}, x_{2}, x_{3}, x_{4}, x_{5}\right)$ satisfying

$$
\begin{cases}x_{0} x_{1}+x_{2} x_{3} & =x_{4}^{2}-\not x_{5}^{2}, \\ \left(a_{1}^{2}-\not \supset a_{3}^{2}\right) x_{3} x_{1}+x_{0} x_{2} & =a_{1} x_{4}^{2}+\not \supset a_{1} x_{5}^{2} .\end{cases}
$$

We count the number of fixed lines. There are two of them inside $\mathbf{P G}(3, q)$. A line outside is incident with a point having coordinates $\left(x_{0}, x_{1}, \ldots, x_{5}\right)$ with $\left(x_{4}, x_{5}\right) \neq(0,0)$. There are $q^{2}-1$ choices to accomplish that for $\left(x_{4}, x_{5}\right)$. Further, in order that the above system of equations has a solution (and hence a unique solution $)$ in $\left(x_{3}, x_{0}\right)$, it is necessary and sufficient that $\left(x_{1}, x_{2}\right) \neq(0,0)$. Again there are $q^{2}-1$ choices to accomplish this. In total, this gives us $\left(q^{2}-1\right)\left(q^{2}-1\right)$ solutions of the above system. Since each line has $q+1$ points (note that such a fixed line outside $\mathbf{P G}(3, q)$ does not intersect $\mathbf{P G}(3, q))$, and each point has $q-1$ different representations with coordinates, we obtain $q^{2}-1$ more fixed lines. Hence in total exactly $q^{2}+1$ of them.

Furthermore, the lines $e_{0} e_{3}$ and $e_{1} e_{2}$ can be regarded as arbitrary fixed lines, and we see that no other fixed line is contained in the space $\left\langle e_{0} e_{3}, e_{1} e_{2}\right\rangle$. This implies that, for two fixed distinct lines $L, M$, we always have that $\{L, M\}^{\perp} \cup\{L, M\}^{\perp \perp}$ contains precisely two fixed lines of $\sigma$. Furthermore, counting the lines of $\mathcal{Q}(5, q)$ thus covered, including the fixed lines (namely, $\frac{\left(q^{2}+1\right) q^{2}}{2} \times((q+1)+(q-1))+\left(q^{2}+1\right)=$ $\left.\left(1+q^{2}\right)\left(1+q^{3}\right)\right)$, we see that every line $X$ which is not fixed by $\sigma$ is in such a set, and hence its image under $\sigma$ is in the same set, implying that $\left\{X, X^{\sigma}\right\}^{\perp \perp} \cup\left\{X, X^{\sigma}\right\}^{\perp}$ meets $S$ in exactly 2 elements.

The lemma is proved.

An involution of $\mathcal{S}$ fixing $\mathcal{S}^{\prime}$ pointwise in the case $q$ odd. We maintain our assumption that $q$ is odd.

Consider in $H^{\prime}$ the group of whorls about both $v_{0}$ and $w_{1}$. This group has even order (see above) and hence contains some nontrivial Sylow 2-subgroup $P_{2}$. Let $\theta$ be an involution of $P_{2}$. Suppose that $\theta$ acts nontrivially on $\mathcal{S}^{\prime}$ (then it fixes exactly $2\left(q^{2}+1\right)$ lines and $q^{2}+3$ points of $\mathcal{S}^{\prime}$, as is easy to show). Consider a point $x$ outside $\mathcal{S}^{\prime}$ on the line $V$. Let $y$ be the unique point on $W$ collinear with $x^{\theta}$ (notice that $V$ and $W$ are fixed). We first claim that $x$ is not fixed under $\theta$. Indeed, if it were, then $\theta$ would fix a subquadrangle of order $\left(s^{\prime}, q^{2}\right), s^{\prime}>1$, containing $v_{0}$ and $w_{1}$. As before, this subquadrangle should coincide with $\mathcal{S}^{\prime}$, contradicting the fact that $x$ does not belong to $\mathcal{S}^{\prime}$. Hence $x$ and $y$ are opposite points and determine a unique subquadrangle $\mathcal{S}(x, y)$ of order $\left(q, q^{2}\right)$ that contains $x, x^{\theta}, y, y^{\theta}$. Notice that $\mathcal{S}\left(x^{\theta}, y^{\theta}\right)=\mathcal{S}(x, y)$. Hence $\mathcal{S}(x, y)$ is stabilized by $\theta$. Suppose that $\theta$ has some fixed point $z$ in $\mathcal{S}(x, y)$. Suppose first that $z$ does not belong to $\mathcal{S}^{\prime}$. Then, as we just proved, $z$ is not incident with any line of $\mathcal{S}^{\prime}$ (any such line would be fixed by $\theta$, and hence would be incident with either $v_{0}$ or $w_{1}$ ). But then the projection of $z$ on any line of $\mathcal{S}^{\prime}$ fixed under $\theta$ is fixed by $\theta$ and does not belong to $\mathcal{S}^{\prime}$, a contradiction. Hence $z$ belongs to $\mathcal{S}^{\prime}$. So it is collinear with either $v_{0}$ or $w_{1}$ (or both). Hence, without loss of generality, we may assume that $v_{0}$ belongs to $\mathcal{S}(x, y)$ (as $\left.v_{0} I V\right)$. But then, since $q$ is odd, a second point $v^{\prime}$ of $\mathcal{S}(x, y)$ on $V$ is also fixed. If it belonged to 
$\mathcal{S}^{\prime}$, then $\mathcal{S}^{\prime}=\mathcal{S}\left(v^{\prime}, \operatorname{proj}_{W} v_{0}\right)=\mathcal{S}(x, y)$, a contradiction, hence it must lie outside $\mathcal{S}^{\prime}$. But this contradicts our previous claim.

So we have shown that $\theta$ induces a fixed point free involution in $\mathcal{S}(x, y)$. Hence there is some line $L$ of $\mathcal{S}(x, y)$ fixed under $\theta$, with $L$ different from $V$ and from $W$ (by Lemma 8.2). This line cannot belong to $\mathcal{S}^{\prime}$, as all the fixed lines in $\mathcal{S}^{\prime}$ are incident with either $v_{0}$ or $w_{1}$, and so $v_{0}$ or $w_{1}$ would belong to $\mathcal{S}(x, y)$ (if distinct lines belong to $\mathcal{S}(x, y)$, then the intersection point also belongs to $\mathcal{S}(x, y)$ ), a contradiction to our previous paragraph. Hence the projection of $v_{0}$ on that line, which is also fixed by $\theta$, is a point on a line of $\mathcal{S}^{\prime}$, and is different from the $q^{2}+3$ fixed points of $\theta$ in $\mathcal{S}^{\prime}$. This is a contradiction to our first claim in the previous paragraph. Hence $\theta$ fixes $\mathcal{S}^{\prime}$ pointwise.

Now the arguments of our previous paragraphs show that $\theta$ does not fix any point outside $\mathcal{S}^{\prime}$. In particular, choosing $x$ as before, the subquadrangle $\mathcal{S}(x, y)$ has no (point) intersection with $\mathcal{S}^{\prime}$. One now easily deduces that we can partition the point set of $V$ into subsets of size $q+1$, each belonging to a subquadrangle of order $\left(q, q^{2}\right)$ which also contains $W$, and which is fixed under $\theta$. This shows that $q+1$ divides $s-q$.

So we may put $s=\frac{1}{2} r\left(q^{3}-q\right)+q$, with $r \in \mathbb{N}$.

The black hole and a final divisibility condition. We finish the case of $q$ odd. We keep our notation of the previous paragraphs.

Denote by $\Omega$ the set of points of $\mathcal{S}$ that are not incident with any line of $\mathcal{S}^{\prime}$. We shall call it for convenience the black hole with respect to $\mathcal{S}^{\prime}$. An easy counting yields $|\Omega|=q^{2}(s-q)\left(s-q^{3}\right)$, and every line not belonging to $\mathcal{S}^{\prime}$ contains exactly $s-q^{3}$ points of $\Omega$.

The collineation $\theta$ still is an involution of $\mathcal{S}$ fixing $\mathcal{S}^{\prime}$ pointwise.

We will now define two classes of subquadrangles of $\mathcal{S}$. The first class consists of all subquadrangles of order $\left(q, q^{2}\right)$ that are (globally) fixed under $\theta$ and that contain at least one point mapped under $\theta$ to a noncollinear point. Such a point necessarily belongs to the black hole. A subquadrangle of order $\left(q, q^{2}\right)$ is uniquely determined by any such point and its image under $\theta$, and has exactly $q^{2}+1$ fixed lines (by Lemma 8.2). The second class of subquadrangles consists of all subquadrangles of order $\left(q, q^{2}\right)$ that are (globally) fixed under $\theta$ and that do not contain any point not collinear with its image. Such subquadrangles have exactly $q^{3}+1$ fixed lines, which form a Hermitian spread of it (again by Lemma 8.2). Hence any quadrangle of the first class shares exactly $q^{2}+1$ lines with $\mathcal{S}^{\prime}$, and every subquadrangle of the second class shares exactly $q^{3}+1$ lines with $\mathcal{S}^{\prime}$. We will now compute the size of the second class.

Consider any point $x$ of a subquadrangle $\mathcal{S}^{\prime \prime}$ of the second class. Then $x^{\theta}$ is collinear with $x$. Take any line $L$ of $\mathcal{S}^{\prime \prime}$ fixed by $\theta$ and different from $x x^{\theta}$. Let $y$ be the projection of $x$ onto $L$. Then $\mathcal{S}^{\prime \prime}$ is uniquely determined by $x$ and $y^{\theta}$. In order to be able to count the number of members of the second class, we have to know precisely in how many cases it happens that, if a point $z$ outside $\mathcal{S}^{\prime}$ is on a line $Z$ of $\mathcal{S}^{\prime}$, and if the point $z^{\prime}$ is the image of the projection of $z$ onto some line of $\mathcal{S}^{\prime}$ opposite $Z$, the subquadrangle of order $\left(q, q^{2}\right)$ containing $z$ and $z^{\prime}$ is in the first class. That is exactly what we will now do.

Let $X$ be an arbitrary line of $\mathcal{S}$ not contained in $\mathcal{S}^{\prime}$. Then there are exactly $q^{3}+1$ lines of $\mathcal{S}^{\prime}$ concurrent with $X$, defining a set $S_{X}$ of $q^{3}+1$ intersection points incident with $X$. Each pair of distinct points $x, y$ of $S_{X}$ now defines a unique subquadrangle 
$\mathcal{S}(x, y)$ of order $\left(q, q^{2}\right)$ as the one containing $x, y, x^{\theta}$ and $y^{\theta}$ (and hence also $X^{\theta}$ ). Each black hole point $b$ on $X$ also defines a unique subquadrangle, namely the one through $b, b^{\theta}$. The latter always belongs to the first class. Also, by Lemma 8.2, each of these has exactly two fixed lines concurrent with both $X$ and $X^{\theta}$. Hence all these subGQ's define a partition of the black hole points on $X$ in sets of size $q-1$. It follows that there are precisely $\frac{s-q^{3}}{q-1}=\frac{1}{2}(r-2) q(q+1)$ of these. Moreover, each subquadrangle having exactly two fixed lines meeting $X$ and $X^{\theta}$, is of the first class and arises in this way. Hence, exactly $\frac{1}{2}(r-2) q(q+1)$ pairs of points of $S_{X}$ give rise to a subquadrangle of the first class, and all other pairs define subquadrangles of the second class. There are $\frac{1}{2}\left(q^{3}\left(q^{3}+1\right)-(r-2) q(q+1)\right)$ pairs remaining. But each subquadrangle they define is counted $\frac{1}{2} q(q+1)$ times (the number of pairs it contains). This gives a total of $q^{2}\left(q^{2}-q+1\right)-r+2$ subquadrangles of the second class containing $X$.

Now there are $q^{2}\left(q^{2}+1\right)(s-q)$ possibilities to choose the line $X$. If we combine (multiply) this with the number of subquadrangles of the second class containing $X$, then we have counted each subquadrangle exactly $q^{2}\left(q^{3}+1\right)$ times (the number of lines not on the fixed Hermitian spread).

So this gives a final total of

$$
\frac{1}{2} r q^{3}\left(q^{2}+1\right)(q-1)-\frac{r(r-2) q\left(q^{2}+1\right)(q-1)}{2\left(q^{2}-q+1\right)}
$$

subquadrangles of the second class.

Since the first term is an integer, the second is, too. As $q^{2}-q+1$ is prime to all of $q, q^{2}+1$ and $q-1$, this implies that $r(r-2)$ is divisible by $q^{2}-q+1$. We know that $r \geq 2$ and $r \leq 2 q-1$. We now show that $r=2$. Suppose first that $r=2 r^{\prime}$ is even. Then $r^{\prime}\left(r^{\prime}-1\right)$ is divisible by $q^{2}-q+1$, and hence $r^{\prime}\left(r^{\prime}-1\right)=0$. Hence $r^{\prime}=1$ and so $s=q^{3}$, implying that $\mathcal{S}$ is dual classical (by [24], Appendix). Suppose now that $r=2 r^{\prime}+1$ is odd. Then $q^{2}-q+1$ divides $4{r^{\prime}}^{2}-1$. Since $r^{\prime}<q$ and $4 r^{\prime 2}-1$ is odd, the only possibilities are $q^{2}-q+1=4{r^{\prime}}^{2}-1$ and $3\left(q^{2}-q+1\right)=4{r^{\prime}}^{2}-1$. In the former case, $q^{2}-q+2$ must be a perfect square, contradicting $(q-1)^{2}<q^{2}-q+2<q^{2}$. In the latter case, $3 q(q-1)=4\left(r^{\prime}-1\right)\left(r^{\prime}+1\right)$. Since $q$ is a power of an odd prime, $q$ divides one of $r^{\prime}-1$ or $r^{\prime}+1$. Put $r^{\prime}=a q \pm 1$; then, since $r^{\prime}<q$, we must have $r^{\prime}=q-1$. But then $3 q(q-1)=4\left(r^{\prime}-1\right)\left(r^{\prime}+1\right)=4 q(q-2)$ implying $q=5, r^{\prime}=4$ and $(s, t)=(545,25)$. But then $s+t$ does not divide $s t(1+s t)$, a contradiction.

This finishes the case of $q$ odd.

We now turn to the case of $q$ even. We will again use some properties of involutions of $\mathcal{Q}(5, q)$.

\section{Some properties of involutions of $\mathcal{Q}(5, q), q$ even.}

Lemma 8.3. Let there be given an involution $\theta$ of $\mathcal{Q}(5, q), q$ even. Then there is at least one fixed point. If $x$ is such a fixed point, and if either all lines through $x$ are fixed, or exactly one line through $x$ is fixed, or all fixed points of $\theta$ collinear with $x$ are incident with one line, then there is a line through $x$ all points of which are fixed.

Proof. Since $(1+q)\left(1+q^{3}\right)$ is odd, $\theta$ fixes at least one point. Let $x$ be such a point. Suppose first that all lines through $x$ are fixed. Since $\theta$ may be viewed as an involution of $\mathbf{P G}(5, q)$, it is clear that, as such, it stabilizes the plane generated by two arbitrary lines through $x$ (remark that $\theta$ has a trivial companion automorphism 
since all lines through $x$ in some 4 -space are fixed). But an involution in a projective plane fixes at least one further point $y$. If $y$ is in $\mathcal{Q}(5, q)$, then $\theta$ fixes all points on the line $x y$ (note that $\theta$ fixes no point of $\mathcal{Q}(5, q)$ not collinear with $x$ ) and the assertion is proved. If $y$ is not in $\mathcal{Q}(5, q)$, then its "polar hyperplane" is fixed, and so there is a subquadrangle of order $(q, q)$ through $x$ fixed. But this subquadrangle is isomorphic to $W(q)$, and hence the points collinear with $x$ are structured as a projective plane, implying that $\theta$ fixes all points on one of the lines through $x$ (keeping in mind that $q$ is even!).

Suppose now that $\theta$ fixes exactly one line through $x$. Let $M$ be a line through $x$ with $M^{\theta} \neq M$. Then the plane $\left\langle M, M^{\theta}\right\rangle$ is fixed by $\theta$ and hence there is at least one fixed point in that plane different from $x$, and evidently not belonging to $\mathcal{Q}(5, q)$. Again this implies that there is a subGQ isomorphic to $W(q)$ through $x$ fixed under $\theta$. Since there is an odd number of lines through $x$ in that subGQ, the fixed line must belong to it. Since no other line through $x$ is fixed, it easily follows that all points on that fixed line $L$ must be fixed themselves (if $x \neq z \mathrm{I} L, z \neq z^{\theta}$, and $L \neq N I z$, then $\left\{N, N^{\theta}\right\}^{\perp \perp}$ contains a fixed line through $x$ ).

Finally suppose that all fixed points of $\theta$ that are collinear with $x$ are incident with one line, say $L$. We may assume that not all lines through $x$ are fixed (as we dealt with that situation above). As before, we obtain a subGQ of order $q$ containing $x$ that is fixed by $\theta$, and our assumption implies, by the foregoing arguments, that it also contains $L$ (otherwise some point collinear with $x$ not on $L$ is fixed). If the companion automorphism of $\theta$ is nontrivial, then $\theta$ fixes points on $\sqrt{q}+1$ lines through $x$, a contradiction. Hence $\theta$ is linear and the result follows as above.

This completes the proof of the lemma.

This now has the following interesting corollary, which we state as a lemma.

Lemma 8.4. If $\theta \neq \mathbf{1}$ is an involution of $\mathcal{S}$, and $s>q^{3}$, then it does not fix any subGQ of order $\left(q, q^{2}\right)$ pointwise.

Proof. Indeed, suppose the involution $\theta$ fixes the subGQ $\mathcal{S}^{\prime}$ pointwise. Note that every fixed point of $\theta$ belongs to $\mathcal{S}^{\prime}$. Suppose $s>q^{3}$, and let $x$ be a black hole point. As in the odd case, there is a unique subquadrangle $\mathcal{S}\left(x, x^{\theta}\right)=\mathcal{S}(x)$ of order $\left(q, q^{2}\right)$ containing $x$ and $x^{\theta} \nsim x$. By Lemma 8.3, there is some fixed point $y$ of $\theta$ in $\mathcal{S}(x)$, necessarily belonging to $\mathcal{S}^{\prime}$. But then $\theta$ fixes all lines through $y$, hence $\theta$ fixes all points in $\mathcal{S}(x)$ on one of the lines $L$ incident with $y$. Hence $\mathcal{S}^{\prime}$ and $\mathcal{S}(x)$ share all points of the line $L$. Projecting $x$ onto $L$, we now see that $x$ lies on a line of $\mathcal{S}^{\prime}$, contradicting the fact that it was supposed to be a black hole point.

Root elations in $\mathcal{S}, q$ even. Consider a nontrivial root-elation of $\mathcal{S}^{\prime}$ fixing all lines concurrent with $V$, and let $\theta$ be a collineation of $\mathcal{S}$ inducing this root-elation in $\mathcal{S}^{\prime}$. Since root-elations in $\mathcal{S}^{\prime}$ have order 2 , the order of $\theta$ is equal to $2 f$, for some natural number $f$. Then $\theta^{f}$ is an involution which, by Lemma 8.4, does not act trivially on $\mathcal{S}^{\prime}$. So $\theta^{f}$ coincides with $\theta$ in $\mathcal{S}^{\prime}$. Hence, without loss of generality, we may assume that $f=1$ and $\theta$ is an involution. Clearly, if $\theta$ fixes a point not on $V$, then it would fix a subGQ of order $\left(q, q^{2}\right)$ pointwise, a contradiction by Lemma 8.4. Hence all fixed points of $\theta$ are incident with $V$ and all fixed lines of $\theta$ meet $V$. Let $u$ be any point on $V$ and suppose that $u$ is not fixed. Then there exists a black hole point $w$ collinear with $u$. Let $\mathcal{S}(w)$ be the subGQ of order $\left(q, q^{2}\right)$ containing $w$ and $w^{\theta}$. It is fixed under $\theta$ and hence has a fixed point. But all of its fixed points must be on $V$, implying by Lemma 8.3 that $V$ belongs to $\mathcal{S}(w)$ and that in particular 
$u$ is fixed. We have shown that $\theta$ fixes all points on $V$. Consequently, there is a group $U\left[v_{0}, v_{1}\right]$ of whorls about both $v_{0}$ and $v_{1}$ with an order divisible by $q$ (in fact, using Lemma 8.4, one can see that there is such a group of order precisely $q$ ). We distinguish two possibilities.

First, suppose all lines concurrent with $V$ are fixed for all appropriate $\theta$. Since $\theta$ belongs to a group of order $q$, we then have that $\left|\{V, W\}^{\perp \perp}\right| \geq q+1$, and, in fact, $\{V, W\}^{\perp \perp}$ contains all lines of $\mathcal{S}^{\prime}$ meeting $v_{0} w_{0}$ and $v_{1} w_{1}$. Since the two latter lines are essentially arbitrary (relative to the next claim), we see that every line outside $\mathcal{S}^{\prime}$ subtends a Hermitian spread of $\mathcal{S}^{\prime}$. Since two Hermitian spreads always share at least one line (as can easily be seen using the dual representation $H\left(3, q^{2}\right)$; there a Hermitian spread is a plane section, and two plane sections meet in a line section, which is always nonempty), there can be no black hole points (since two lines through such a point subtend disjoint spreads). Hence $s=q^{3}$ and $\mathcal{S}$ is dual classical by [24], Appendix B.

Next, suppose that not all lines concurrent with $V$ are fixed by some $\theta$. We claim that we may assume that $s$ is divisible by $q^{3}$.

Indeed, let $u$ be a point on $V$ incident with a line $L$ not fixed by $\theta$. We remark that, by the construction of $\theta$, the latter acts semi-regularly on the set of lines through $u$ different from $V$ (otherwise, since not all lines through $u$ are fixed, $\theta$ induces a semi-linear collineation in the appropriate subquadrangle, implying that $\theta$ fixes at least one point not on $V$, a contradiction). Let $\mathcal{S}^{\prime \prime}$ be the subGQ of order $\left(q, q^{2}\right)$ containing $u$ and $w_{0}$. (So $u \nsim w_{0}$.) By conjugating with torus-elements (i.e., collineations of $\mathcal{S}^{\prime \prime}$ fixing $u, V, W$ and $w_{0}$ ), we see that the group $K$ of whorls about $v_{0}$ which are dual whorls about $V$ acts transitively on the set $\mathcal{L}_{u}$ of lines through $u$ distinct from $V$ itself; hence its order is divisible by $q^{2}$. The action of $K$ on $\mathcal{L}_{u}$ clearly coincides with the "translation group" inherited from $\mathcal{S}^{\prime \prime}$ (or from any subGQ of order $\left(q, q^{2}\right)$ through $u$, as follows immediately from the main hypothesis that $u$ is a center of transitivity). Suppose now that $K$ does not act semi-regularly on the points of $v_{0} w_{0}$ different from $v_{0}$. Then some nontrivial element $\psi$ of $K$ fixes some point, say, $w_{0}$ (without loss of generality). Clearly $\psi$ cannot fix all lines through $u$. Also, $\psi$ is an involution because $\psi^{2}$ fixes all lines through $u$. Using conjugation with respect to the same torus elements as above, we see that we may assume that $K$ fixes $w_{0}$. By the transitivity, there is a group $K^{\prime}$ of whorls about $v_{0}$, fixing $v_{0} w_{0}$ pointwise, fixing $u$, and acting regularly on $\mathcal{L}_{u}$. Let $\varphi \in K^{\prime}$ be arbitrary; then the commutator $[\psi, \varphi]$ is either a root-elation and belongs to $K$ (and then $\mathcal{S}$ is half Moufang because all nontrivial elements of $K$ are conjugate by the foregoing torus elements) or is trivial. In the trivial case, the product $\varphi \psi$ (where we now choose $\varphi$ in such a way that it has the same action on $W$ as $\psi$ ) is an involution fixing $\mathcal{S}^{\prime \prime}$ pointwise, hence the identity, hence again $\psi$ belongs to $K \cap K^{\prime}$ and $\mathcal{S}$ is half Moufang by considering conjugation with the torus elements.

Hence we may assume that $K$ acts semi-regularly on the points of $v_{0} w_{0}$ different from $v_{0}$. Likewise, we may assume that the group generated by $K$ and $U\left[v_{0}, u\right]$ acts semi-regularly on the points of $v_{0} w_{0}$ different from $v_{0}$. Since that group is easily seen to have an order divisible by $q^{3}$, the claim follows. We deduce that, since we already know that $q-1$ is a divisor of $s-q$, the number $s$ can be written as $s=r q^{3}(q-1)+q^{3}$. But we know that $s \leq q^{4}$, so $r=1$ and $s=q^{4}$.

The case $s=q^{4}$. Now suppose that $s=q^{4}=t^{2}$. Then by 1.2.4 of FGQ, each triad of lines has $q^{2}+1$ centers. Now fix an arbitrary point $x$ of $\mathcal{S}$, and consider 
the group $G$ of all whorls about $x$. Let $y \not x$. If $\mathbf{1} \neq \theta \in G_{y}$ and $\theta$ is an involution, then $\theta$ fixes a subGQ of order $\left(q, q^{2}\right)$ pointwise, a contradiction by Lemma 8.4, so the order of $\theta$ is odd. It follows that a Sylow 2 -subgroup $G_{2}$ of $G$ has size $q^{10}$, and acts regularly on the points not collinear with $x$. So $\mathcal{S}$ is an EGQ with elation point $x$, and the elation group is a 2-group. As all spans of noncollinear points have size 2, it follows by Proposition 5.5 that there must be nonconcurrent line spans of size at least 3 . But this contradicts the fact that each triad of lines has precisely $q^{2}+1$ centers.

This completes the proof of Theorem B.

\section{ACKNOWLEDGEMENT}

Part of this research was done while the first author was a Research Fellow supported by the Flemish Institute for the Promotion of Scientific and Technological Research in Industry (IWT), grant no. IWT/SB/991254/Thas.

\section{REFERENCES}

[1] I. Bloemen, J. A. Thas and H. Van Maldeghem. Elation generalized quadrangles of order $(p, t), p$ prime, are classical, J. Statist. Plann. Inference 56 (1996), 49-55. MR1435520 (98c:51007)

[2] R. C. Bose And S. S. Shrikhande. Geometric and pseudo-geometric graphs $\left(q^{2}+1, q+1,1\right)$, J. Geom. 2/1 (1972), 75-94. MR0302468 (46:1612)

[3] A. E. Brouwer. The complement of a geometric hyperplane in a generalized quadrangle is usually connected, Finite Geometry and Combinatorics, Edited by F. De Clerck et al., Cambridge University Press, London Math. Soc. Lect. Notes Ser. 191 (1993), 53-57. MR1256263 (94m:51005)

[4] F. Buekenhout and H. Van Maldeghem. Finite distance transitive generalized polygons, Geom. Dedicata 52 (1994), 41-51. MR1296145 (95g:51006)

[5] P. Fong And G. M. Seitz. Groups with a (B,N)-pair of rank 2, I, Invent. Math. 21 (1973), 1-57. MR0354858 (50:7335)

[6] P. Fong And G. M. Seitz. Groups with a (B,N)-pair of rank 2, II, Invent. Math. 24 (1974), 191-239. MR0360796 (50:13243)

[7] D. Frohardt. Groups which produce generalized quadrangles, J. Combin. Theory Ser. A 48 (1988), 139-145. MR938864 (89d:51015)

[8] C. Hering, W. M. Kantor And G. M. Seitz. Finite groups with a split BN-pair of rank 1, I, J. Algebra 20 (1972), 435-475. MR0301085 (46:243)

[9] D. G. Higman. Partial geometries, generalized quadrangles and strongly regular graphs, Atti Covegno di Geometria e Sue Applicazioni, Edited by A. Barlotti, Perugia (1971), 263-293. MR0366698 (51:2945)

[10] D. G. Higman. Invariant relations, coherent configurations and generalized polygons, Combinatorics, Part 3, Edited by M. Hall and J. H. Van Lint, Math. Centre Tracts 57, Amsterdam (1974), 27-43. MR0379244 (52:150)

[11] J. W. P. Hirschfeld. Projective Geometries over Finite Fields, Oxford Mathematical Monographs, The Clarendon Press, Oxford University Press, New York, 1979. MR554919 (81h:51007)

[12] B. Huppert. Endliche Gruppen I, Springer-Verlag, Berlin, 1967. MR0224703 (37:302)

[13] W. M. Kantor. Automorphism groups of some generalized quadrangles, in: Adv. Finite Geom. and Designs, Proceedings Third Isle of Thorn Conference on Finite Geometries and Designs, Brighton 1990, Edited by J. W. P. Hirschfeld et al., Oxford University Press, Oxford (1991), 251-256. MR1138747 (92m:51009)

[14] D. Passman. Permutation Groups, Benjamin, New York, Amsterdam, 1968. MR0237627 (38:5908)

[15] S. E. PAyne. Finite generalized quadrangles: a survey, Proceedings of the International Conference on Projective Planes (Washington State Univ., Pullman, Wash., 1973), pp. 219-261, Washington State Univ. Press, Pullman, Wash., 1973. MR0363954 (51:209) 
[16] S. E. PAyne. Collineations of finite generalized quadrangles, Finite Geometries, Lecture Notes in Pure and Appl. Math. 82, Dekker, New York (1983), 361-390. MR690820 (84d:51022)

[17] S. E. Payne And J. A. Thas. Finite Generalized Quadrangles, Research Notes in Mathematics 110, Pitman Advanced Publishing Program, Boston/London/Melbourne, 1984. MR767454 (86a:51029)

[18] G. M. Seitz. Flag-transitive subgroups of Chevalley groups, Ann. of Math. 97 (1973), 27-56. MR0340446 (49:5201)

[19] E. Shult. On a class of doubly transitive groups, Illinois J. Math. 16 (1972), 434-455. MR0296150 (45:5211)

[20] K. Tent and H. Van Maldeghem. Moufang polygons and irreducible spherical BN-pairs of rank 2, I, Adv. Math. 174 (2003), 254-265. MR1963695 (2004b:20045)

[21] J. A. Thas. Characterizations of generalized quadrangles by generalized homologies, J. Combin. Theory Ser. A 40 (1985), 331-341. MR814418 (87a:51019)

[22] J. A. Thas. The classification of all $(x, y)$-transitive generalized quadrangles, J. Combin. Theory Ser. A 42 (1986), 154-157. MR843472 (87k:51035)

[23] J. A. Thas. Generalized quadrangles and the theorem of Fong and Seitz on BN-pairs, Preprint.

[24] J. A. Thas and H. Van Maldeghem. Lax embeddings of generalized quadrangles in finite projective spaces, Proc. London Math. Soc. 82 (2001), 402-440. MR1806877 (2002b:51004)

[25] J. A. Thas, S. E. Payne and H. Van Maldeghem. Half Moufang implies Moufang for finite generalized quadrangles, Invent. Math. 105 (1991), 153-156. MR1109623 (92e:51009)

[26] K. ThAs. Strong Elation Generalized Quadrangles, I and II, Lectures given at Ghent University Incidence Geometry Seminar, 1999.

[27] K. Thas. On symmetries and translation generalized quadrangles, in: Developments in Mathematics 3, Proceedings of the Fourth Isle of Thorns Conference Finite Geometries, 16-21 July 2000, Edited by A. Blokuis et al., Kluwer Academic Publishers (2001), 333-345. MR2061813 (2005b:51012)

[28] K. Thas. Automorphisms and Characterizations of Finite Generalized Quadrangles, in: Generalized Polygons, Proceedings of the Academy Contact Forum 'Generalized Polygons', 20 October 2000, Palace of the Academies, Brussels (2001), 111-172.

[29] K. Thas. A theorem concerning nets arising from generalized quadrangles with a regular point, Des. Codes Cryptogr. 25 (2002), 247-253. MR1900771 (2003e:51008)

[30] K. Thas. Classification of span-symmetric generalized quadrangles of order s, Adv. Geom. 2 (2002), 189-196. MR1895346 (2003f:51015)

[31] K. Thas. The classification of generalized quadrangles with two translation points, Beiträge Algebra Geom. 43 (2002), 365-398. MR1957745 (2003k:51005)

[32] K. Thas. Automorphisms and Combinatorics of Finite Generalized Quadrangles, Ph.D. Thesis, Ghent University, March 2002.

[33] K. Thas. Symmetry in Finite Generalized Quadrangles, Birkhäuser Verlag, Frontiers in Mathematics 1, Basel, Boston, Berlin, 2004. MR2036432 (2005e:51006)

[34] J. Tits. Sur la trialité et certains groupes qui s'en déduisent, Inst. Hautes Etudes Sci. Publ. Math. 2 (1959), 13-60.

[35] J. Tits. Les groupes simples de Suzuki et de Ree, Séminaire Bourbaki 13(210) (1960), 1 - 18.

[36] J. Tits. Théorème de Bruhat et sous-groupes paraboliques, C. R. Acad. Sci. Paris Ser. I. Math. 254 (1962), 2910-2912. MR0138706 (25:2149)

[37] J. Tits. Buildings of Spherical Type and Finite BN-Pairs, Lecture Notes in Mathematics 386, Springer, Berlin, 1974. MR0470099 (57:9866)

[38] J. Tits. Classification of buildings of spherical type and Moufang polygons: a survey, Coll. Intern. Teorie Combin. Acc. Naz. Lincei, Roma 1973, Atti dei convegni Lincei 17 (1976), 229-246. MR0444793 (56:3140)

[39] J. TiTs. Twin buildings and groups of Kac-Moody type, Proceedings of a Conference on Groups, Combinatorics and Geometry, Edited by M. Liebeck and J. Saxl, Durhum 1990, London Math. Soc. Lecture Note Ser. 165, Cambridge University Press, Cambridge (1992), 249-286. MR1200265 (94d:20030)

[40] J. Tits and R. Weiss. Moufang Polygons, Springer Monographs in Mathematics, SpringerVerlag, Berlin, 2002. MR1938841 (2003m:51008)

[41] H. Van Maldeghem. A geometric characterization of the perfect Suzuki-Tits ovoids, J. Geom. 58 (1997), 192-202. MR1434192 (98b:51006) 
[42] H. Van Maldeghem. Some consequences of a result of Brouwer, Ars Combin. 48 (1998), 185-190. MR1623011 (99d:51005)

[43] H. Van Maldeghem. Generalized Polygons, Birkhäuser, Monographs in Mathematics 93, Basel, Boston, Berlin, 1998. MR1725957 (2000k:51004)

Department of Pure Mathematics and Computer Algebra, Ghent University, GalGlaAn 2, B-9000 Ghent, Belgium

E-mail address: kthas@cage.UGent.be

Department of Pure Mathematics and Computer Algebra, Ghent University, GalGlaAn 2, B-9000 Ghent, Belgium

E-mail address: hvm@cage.UGent.be 